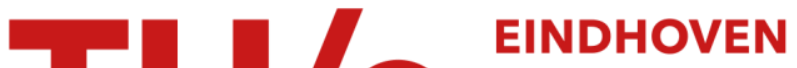 UNIVERSITY OF TECHNOLOGY
}

\section{Dynamic demand fulfillment in spare parts networks with multiple customer classes}

Citation for published version (APA):

Tiemessen, H. G. H., Fleischmann, M., Houtum, van, G. J. J. A. N., van Nunen, J. A. E. E., \& Pratsini, E. (2012). Dynamic demand fulfillment in spare parts networks with multiple customer classes. (BETA publicatie : working papers; Vol. 377). Technische Universiteit Eindhoven.

Document status and date:

Published: 01/01/2012

\section{Document Version:}

Publisher's PDF, also known as Version of Record (includes final page, issue and volume numbers)

\section{Please check the document version of this publication:}

- A submitted manuscript is the version of the article upon submission and before peer-review. There can be important differences between the submitted version and the official published version of record. People interested in the research are advised to contact the author for the final version of the publication, or visit the $\mathrm{DOI}$ to the publisher's website.

- The final author version and the galley proof are versions of the publication after peer review.

- The final published version features the final layout of the paper including the volume, issue and page numbers.

Link to publication

\section{General rights}

Copyright and moral rights for the publications made accessible in the public portal are retained by the authors and/or other copyright owners and it is a condition of accessing publications that users recognise and abide by the legal requirements associated with these rights.

- Users may download and print one copy of any publication from the public portal for the purpose of private study or research.

- You may not further distribute the material or use it for any profit-making activity or commercial gain

- You may freely distribute the URL identifying the publication in the public portal.

If the publication is distributed under the terms of Article 25fa of the Dutch Copyright Act, indicated by the "Taverne" license above, please follow below link for the End User Agreement:

www.tue.nl/taverne

Take down policy

If you believe that this document breaches copyright please contact us at:

openaccess@tue.nl

providing details and we will investigate your claim. 


\section{Beta}

Research School for Operations

Management and Logistics

Dynamic demand fulfillment in spare parts networks with multiple customer classes

H.G.H. Tiemessen, M. Fleischmann, G.J. van Houtum,

J.A.E.E. van Nunen, E. Pratsini

Beta Working Paper series 377

\begin{tabular}{|rl|}
\hline BETA publicatie & WP 377 (working \\
& paper) \\
ISBN & \\
ISSN & \\
NUR & 804 \\
Eindhoven & March 2012 \\
\hline
\end{tabular}




\title{
Dynamic demand fulfillment in spare parts networks with multiple customer classes
}

\author{
H.G.H. Tiemessen ${ }^{\mathrm{a}, *}$, M. Fleischmann ${ }^{\mathrm{b}}$, G.J. van Houtum ${ }^{\mathrm{c}}$, J.A.E.E. van Nunen ${ }^{\mathrm{d}}$, E. Pratsini ${ }^{\mathrm{a}}$ \\ ${ }^{a}$ Department of Mathematical and Computational Sciences, IBM Research, Zurich Research Laboratory, 8803 Rüschlikon, \\ Switzerland \\ ${ }^{b}$ Business School, University of Mannheim, P.O. Box 1034 62, 68131 Mannheim, Germany \\ ${ }^{c}$ School of Industrial Engineering, Eindhoven University of Technology, P.O. Box 513, 5600 MB Eindhoven, the Netherlands \\ ${ }^{d}$ Rotterdam School of Management, Erasmus University, P.O. Box 1738, 3000 DR Rotterdam, the Netherlands
}

\begin{abstract}
We study real-time demand fulfillment for networks consisting of multiple local warehouses, where spare parts of expensive technical systems are kept on stock for customers with different service contracts. Each service contract specifies a maximum response time in case of a failure and hourly penalty costs for contract violations. Part requests can be fulfilled from multiple local warehouses via a regular delivery, or from an external source with ample capacity via an expensive emergency delivery. The objective is to minimize delivery cost and penalty cost by smartly allocating items from the available network stock to arriving part requests. We propose a dynamic allocation rule that belongs to the class of one-step lookahead policies. To approximate the optimal relative cost, we develop an iterative calculation scheme that estimates the expected total cost over an infinite time horizon, assuming that future demands are fulfilled according to a simple static allocation rule. In a series of numerical experiments, we compare our dynamic allocation rule with the optimal allocation rule, and a simple but widely used static allocation rule. We show that the dynamic allocation rule has a small optimality gap and that it achieves an average cost reduction of $7.9 \%$ compared to the static allocation rule on a large test bed containing problem instances of real-life size.

Keywords: Inventory, Spare parts, Dynamic demand fulfillment, Multiple customer classes
\end{abstract}

\section{Introduction}

We consider demand fulfillment for networks consisting of multiple local warehouses, where spare parts of expensive technical systems are kept on stock. Downtime costs (like opportunity cost in case of lost production, liability cost, or loss of goodwill) are usually high, and can easily run into thousands of euros per hour. Therefore, it is important that the availability of the systems is high and that down-situations are

\footnotetext{
${ }^{*}$ Corresponding author. Tel.: +414153429 59; fax: +41447248964

Email addresses: hti@zurich.ibm.com (H.G.H. Tiemessen), MFleischmann@bwl .uni-mannheim.de (M. Fleischmann), g.j.v.houtum@tue.nl (G.J. van Houtum), pra@zurich.ibm.com (E. Pratsini)
} 
recovered quickly. Original Equipment Manufacturers (OEMs) of high-tech equipment usually sell their equipment with a variety of service contracts at different prices. Typically, these service contracts commit to maintenance service within 2 hours, 4 hours, 8 hours, or the next, or second next business day. Due to strong fluctuations in spare parts demand and strict service deadlines, spare parts logistics execution must be responsive. This is achieved by means of fast call-handling, accurate (remote-) problem diagnosis, 24 hours operations, fast transportation modes, and stocking locations at close distance to the customer site.

OEMs often serve customers with different service deadlines from the same network. Because of the pooling effect, this requires (much) lower investments in inventory than operating separate networks per contract type. To serve customers with premium contracts in time, OEMs must establish dense same day networks of stocking locations. Consequently, many customers can be served within the service deadline from multiple stocking locations.

A major challenge for the OEM is to minimize inventory holding cost, replenishment cost and fulfillment cost while providing the promised service to its customers. OEMs can simultaneously influence cost and customer service in two possible ways: (i) through calculating appropriate base stock levels for all warehouses, and (ii) through determining an appropriate allocation rule for selecting the warehouse that is used to fulfill a real-time spare parts demand. Whereas the calculation of base stock levels is a tactical planning problem where decisions are usually taken every 3-6 months, the calculation of a stock allocation rule is an operational planning problem where usually many decisions must be taken each day. In this study we focus on the second problem and assume that the base stock levels are given.

In practice, we see that many OEMs have implemented a simple static allocation rule that fulfills a real-time spare parts demand from the closest warehouse with stock at hand. See Reijnen et al. (2009), and Kranenburg \& Van Houtum (2009) for case studies at OEMs of high tech equipment. This allocation rule is popular because it only requires static time/distance information and the set of warehouses with stock at hand. One drawback of this rule is that it does not use some potentially valuable information for making smart allocation decisions: real-time stock level information, and demand forecast information. Another drawback of this rule is that it does not consider customer base heterogeneity. As a result, each customer, despite generating different revenues to the OEM, receives a similar treatment in terms of customer service. This is unattractive for the OEM for two reasons: First, customers who bought expensive high-end contracts might consider this unfair, and this might disturb the customer relationship. Second, high base stock levels are needed to provide the promised service to customers with tight service deadlines. It is thus attractive to differentiate between the customers based on their service contract. Customer differentiation can be realized using critical levels. For single location models this is often the only choice. In networks however, customer differentiation can also be realized through dynamic allocation. In this paper we explore this direction. 
Our goal in this paper is to investigate the benefits of using real-time stock level information and demand forecast information for real-time demand management in spare parts inventory networks. We summarize our problem setting as follows: We consider a single item, single echelon, multi location inventory network where spare parts are kept on stock for customers with different service contracts. Each service contract specifies a maximum response time in case of a failure, and contract violations are penalized. The objective is to minimize the sum of delivery cost and penalty cost by smartly allocating items from the available network stock to arriving part requests. What makes this task challenging is that the choice to fulfill a part request from a particular source location does not only cause a certain direct cost, but also impacts the opportunities to fulfill demands in the near future (at least until the allocated item is replenished again). We present an average cost Markov Decision Process (MDP) formulation of this problem, which enables us to compute the optimal allocation rule for small problem instances. To handle problem instances of reallife size, we develop a one-step lookahead policy where we approximate the optimal relative cost with an estimate of the relative cost under a simple static allocation rule.

In a series of numerical experiments, we compare the performance of the proposed allocation rule with the optimal allocation rule and two benchmark allocation rules.

To summarize, the paper makes the following contributions:

- We develop a dynamic allocation rule that is applicable to problem instances of real-life size. This allocation rule is a one-step lookahead policy that takes into account base stock levels, actual stock levels, and demand forecast information. We show that the optimality gap of the proposed allocation rule is usually small (i.e. average gap is less than $2 \%$ ).

- We characterize our dynamic allocation rule by comparing its allocation decisions to the allocation decisions of a simple static allocation rule. We show that the dynamic allocation rule achieves considerable cost savings by deviating from the simple static allocation rule in a relatively small number of situations. In particular, we show that the dynamic allocation rule is more reluctant to take away the last item at a local warehouse and less reluctant to use emergency deliveries from the central warehouse.

- We show that dynamic allocation leads to significant cost savings compared to a simple but widely used static allocation rule by numerical experiments on a test bed that is inspired by IBM's spare parts network in Europe. We illustrate the impact of key problem characteristics on the potential benefits.

The paper is structured as follows. We start with a literature review and position our research in Section 2. In Section 3, we describe our model and discuss important assumptions. In Section 4, we introduce our new dynamic allocation rule. Section 5 presents a numerical study that compares the proposed dynamic 
allocation rule with the optimal allocation rule, and two benchmark allocation rules. Finally, in Section 6, we summarize our results and draw conclusions.

\section{Literature review}

Our work contributes to a rich literature on spare parts inventory models. The literature that is most related to the work in this paper, comes from three streams.

In the first stream of literature, inventory models with fixed lateral transshipment rules are studied. In this stream, demand occurring at a local warehouse with no stock at hand can be fulfilled via a stock transfer from another local warehouse. In literature, models are developed for evaluating system performance, either exactly or approximately. An important contribution in this stream is made by Axsäter (1990) who studied a two echelon model with one central warehouse and a number of local warehouses. In his model demand is fulfilled from the local warehouse whenever possible. If no items are available, an item is sent from a randomly chosen local warehouse with stock at hand. Demand rates observed by each warehouse are approximated by assuming that all demand streams are Poisson. Based on the resulting set of equations, he provides an iterative algorithm to obtain steady state probabilities. In the spirit of Axsäter (1990), Kukreja et al. (2001), Wong et al. (2005), Kutanoglu (2008), Kranenburg \& Van Houtum (2009), and Reijnen et al. (2009) develop different models for evaluating and optimizing system performance under given allocation rules. All of these models assume static allocation rules whereas we focus on dynamic allocation rules.

The second stream of literature also studies inventory models with lateral transshipments, but the lateral transshipment rule is now subject to optimization. An important paper in this stream is Axsäter (2003). The author considers a backordering model where a particular local warehouse with no stock at hand receives a customer demand. The task is to select a warehouse to fulfill the demand. The impact of all possible sourcing decisions on the total cost is approximated by considering the direct cost and the additional future cost associated with a temporary reduction of the stock level at the source location. Future cost is calculated under the assumption that no lateral transshipments will take place. Minner et al. (2003) consider a similar model in a retail environment with lost sales. Another interesting contribution in this stream is made by van Van Wijk et al. (2011) who provide an exact analysis for a two location setting with given base stock policies and exponential lead times. What differentiates our work from this stream, is that we consider inventory networks where part requests can be fulfilled directly (i.e. without lateral transshipment) from multiple local warehouses in the network, and that we support multiple customer classes. For a recent and comprehensive literature review on lateral transshipments we refer to Paterson et al. (2011).

The third stream of research that is relevant for our work studies inventory rationing and revenue management. Inventory rationing techniques support the allocation of inventory units among a heterogeneous 
customer base, by setting critical inventory levels and/or setting inventory control mechanisms. The concept of critical levels was introduced by Veinott Jr (1965) and Topkis (1968) and since then, solutions have been provided for various control policies and demand classes. For a comprehensive literature review on inventory rationing we refer to Teunter \& Klein Haneveld (2008). Although the concept of critical levels supports customer heterogeneity, it cannot easily be applied to our problem because of two reasons. First, critical level literature usually assumes a single source location. Sourcing flexibility - a key characteristic of our problem - is not accounted for. Second, the concept of critical levels offers only limited opportunities for customer differentiation in spare parts settings like ours where stock levels are low and the number of customer classes is usually more than two.

To overcome these difficulties, Jalil (2011) follows the concepts of revenue management to formulate a problem similar to ours as a multi-period MDP. The relative value function is approximated using linear programming. In the linear program, remaining stock at the end of the horizon has no value, future replenishments are ignored, and demand is assumed to be deterministic. In numerical experiments, he shows that in situations where customer heterogeneities are high and actual stock levels are low the revenue management heuristic achieves significant lower total cost over the next 25 time periods than the static allocation rule. What differentiates our work from Jalil (2011) is that we approximate the relative value function using an infinite time horizon taking into account future replenishments and honoring the stochastic nature of demand.

\section{Problem description and model formulation}

In this section we define the inventory control problem, discuss assumptions, introduce our notation, and formulate our model.

\subsection{Problem description and notation}

We consider a single item, single echelon, spare parts inventory network consisting of multiple local warehouses where spare parts are kept on stock for customers with different service contracts. Whenever a part at a customer site fails, it has to be replaced by a spare part. Part requests can be fulfilled from every warehouse in the network with stock at hand. The central warehouse keeps spare parts to replenish the local warehouses, and has also access to an emergency delivery mode to fulfill customer demand. The delivery time and the delivery cost from a warehouse to a customer are fixed and depend on the geographical coordinates and the delivery mode (emergency or regular). Typically, emergency deliveries are significantly more expensive than regular deliveries. Customers have service contracts with committed response and repair targets backed by penalties in case of contract violations. Penalty cost grows linearly in the delivery 
time beyond the service deadline, and depend on the contract type. Typically, the hourly penalty cost rate for 2 hours service contracts is (much) higher than for 8 hours contracts. Our objective is to minimize the sum of the average annual delivery cost and the average annual penalty cost.

\subsection{Discussion of main assumptions}

(i) The central warehouse has ample stock. The reason for this assumption is that replenishment decisions at the central warehouses are often decoupled from replenishment and allocation decisions at lower network echelons. In real life, we often see that the central warehouse can obtain new items from multiple channels such as regular suppliers, emergency suppliers, repair, and assembly/production facilities.

(ii) Part requests from each customer follow an independent Poisson process. The Poisson assumption is common in spare parts logistics.

(iii) Replenishment lead times are exponentially and identically distributed and have the same mean for all local warehouses. The assumption of equal replenishment lead times is justified because in reallife replenishment lead times are simply fixed at the same values for all local warehouses in the same geographical region. The assumption of an exponential shape of the replenishment lead time distribution is made to facilitate an exact MDP analysis for problem instances of sufficiently small size. Alfredsson \& Verrijdt (1999) showed that the performance of a model with a static allocation rule in a complete pooling situations is rather insensitive to the choice of exponential or constant replenishment lead times. It is reasonable to assume that this also holds for our model. However, it is also clear that in situations with constant replenishment lead times, information on remaining replenishment lead times can be useful to calculate smart allocation decisions. We leave this for further research.

(iv) Inventories at local warehouses are controlled through continuous-time base stock policies with given base stock levels. In real life, stock levels are often reviewed only once a day. Since the replenishment lead time for a local warehouse is typically 3-6 days, we can however accurately approximate the periodic replenishment with a continuous replenishment with an adjusted average replenishment lead time. The assumption of base stock control at the local warehouses is justified because we consider inventory networks of expensive technical parts where holding cost is usually high and demand is usually low. Finally, we assume that the base stock levels are given because we want to focus on operational planning. In real life, base stock levels are often calculated every 3-6 months from multi-item models. These multi-item models may, among other things, incorporate demand forecast information, target time-based fill rates for part groups, and available stocking space. 
(v) Service deadlines, penalty cost, and delivery times are such that is never beneficial to backorder demand. Demand is thus always fulfilled immediately, either from a local warehouse or from the central warehouse (by means of an emergency delivery).

(vi) Customers in the same geographical area are aggregated into one customer region. Delivery times and delivery costs for all customers in the same customer region are the same. Demand rates for spare parts are available for each pair of customer region and contract type. This assumption makes sense because the expected life time of a spare part is large, and accurate information on the number and the condition of installed parts is usually not available. Hence, demand rate estimates are much more reliable on customer region level than on individual customer level.

The notation of our model is given in Table 1 .

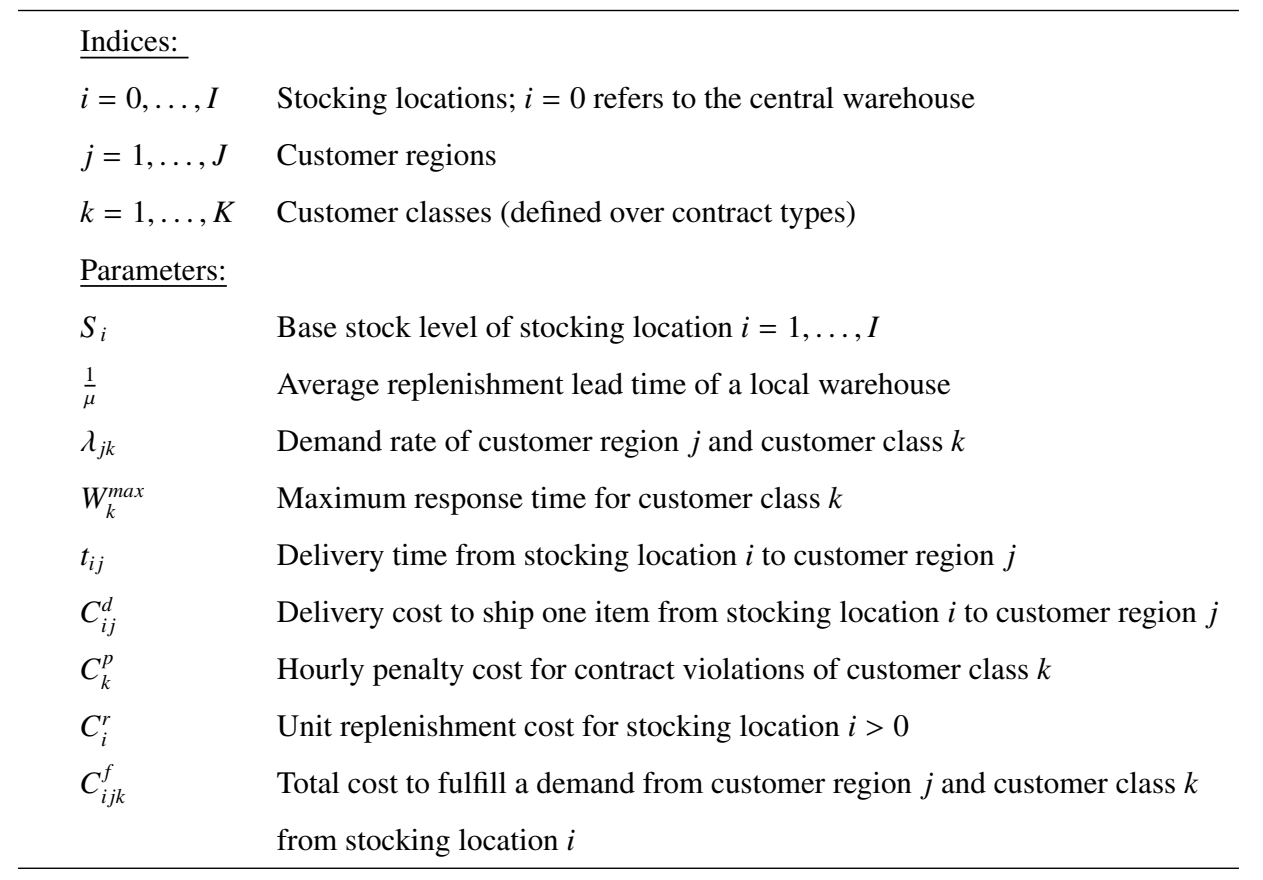

Table 1: Notation

Because we assume one-for-one replenishment at all local warehouses, each spare part demand leads to one spare part leaving the central warehouse (either directly as an emergency delivery to the customer or as a replenishment shipment to the local warehouse that fulfills the customer demand). Consequently, replenishment cost at the central warehouse can be ignored because it does not depend on the allocation decision. Together with assumption (v) this implies that we can pre-calculate the total cost for fulfilling a part request from customer region $j$ and customer class $k$ from stocking location $i$ according to:

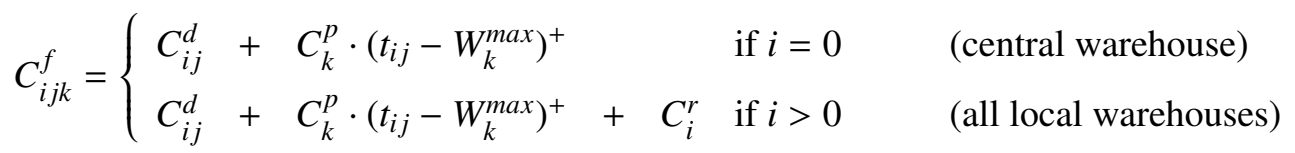




\subsection{MDP formulation}

In this section we formulate the real-time allocation problem as a continuous-time average cost MDP (see e.g. Bertsekas (2007), p.310-316). State transitions and action selections take place at time instances when one of the following two event types occurs: (i) a replenishment order arrives at a local warehouse, or (ii) a customer issues a new part request. Times between successive transitions have an exponential probability distribution.

We describe the state of the system by $\mathbf{x}=(\mathbf{z}, j, k)$, with $\mathbf{z}=\left(z_{1}, \ldots, z_{I}\right)$ the $I$-dimensional vector of actual stock levels at the local warehouses, and $(j, k)$ references to the customer region and the customer class associated with the arriving part request. If the state refers to a replenishment order arrival, we set $j$ and $k$ equal to 0 . We define the state space $\mathcal{S}$ as $\mathcal{S}=\left\{\left(\left(z_{1}, \ldots, z_{I}\right), j, k\right) \mid z_{i} \in\left\{0, \ldots, S_{i}\right\}, i=1, \ldots, I, j=\right.$ $1, \ldots, J, k=1, \ldots, K\}$.

We now define the action space $\mathcal{A}$, and the set of admissible actions $\mathcal{A}(\mathbf{x})$ for each state $\mathbf{x} \in \mathcal{S}$. The action space for our model is $\mathcal{A}=\{-1,0, \ldots, I\}$. Here, action $i \geq 0$ stands for the decision to send a spare part from warehouse $i$ to the customer who has just issued a part request, and action -1 stands for the decision to do nothing. Obviously, we have that $\mathcal{A}(\mathbf{z}, 0,0)=\{-1\}$ for all $\mathbf{z}$. The set of admissible actions for a state that represents a demand arrival, consists of all local warehouses with stock at hand plus the central warehouse: $\mathcal{A}(\mathbf{z}, j, k)=\left\{i \mid z_{i}>0, i=1, \ldots, I\right\} \cup\{0\}$ for all $(\mathbf{z}, j, k) \in \mathcal{S}$ with $j, k>0$.

Next, we describe the transition rates and the transition probabilities for our MDP formulation. We define $\mathbf{e}_{i}$ for $i>0$ as the $I$-dimensional unit vector with a 1 at position $i$ and $\mathbf{e}_{0}$ as the zero vector, i.e. $\mathbf{e}_{0}=\mathbf{0}$.

Transition type 1: initial state: $\mathbf{x}=(\mathbf{z}, 0,0)$, action: -1 , next event: part request from customer region $n$ and customer class $p$, next state: $\mathbf{y}=(\mathbf{z}, n, p)$. The transition rate is $\lambda_{n p}$ and the transition probability $p_{\mathbf{x}, \mathbf{y}}(-1)$ is equal to $\lambda_{n p} /\left[\sum_{t=1}^{J} \sum_{u=1}^{K} \lambda_{t u}+\mu \sum_{r=1}^{I}\left(S_{r}-z_{r}\right)\right]$.

Transition type 2: initial state: $\mathbf{x}=(\mathbf{z}, 0,0)$, action: -1 , next event: order arrival at local warehouse $m$, next state: $\mathbf{y}=\left(\mathbf{z}+\mathbf{e}_{m}, 0,0\right)$. The transition rate is $\mu\left(S_{m}-z_{m}\right)$ and the transition probability $p_{\mathbf{x}, \mathbf{y}}(-1)$ is equal to $\mu\left(S_{m}-z_{m}\right) /\left[\sum_{t=1}^{J} \sum_{u=1}^{K} \lambda_{t u}+\mu \sum_{r=1}^{I}\left(S_{r}-z_{r}\right)\right]$.

Transition type 3-a: initial state: $\mathbf{x}=(\mathbf{z}, j, k)$ with $(j, k) \in\{1, \ldots, J\} \times\{1, \ldots, K\}$, action: 0 , next event: part request from customer region $n$ and customer class $p$, next state: $\mathbf{y}=(\mathbf{z}, n, p)$. The transition rate is $\lambda_{n p}$ and the transition probability $p_{\mathbf{x}, \mathbf{y}}(0)$ is equal to $\lambda_{n p} /\left[\sum_{t=1}^{J} \sum_{u=1}^{K} \lambda_{t u}+\mu \sum_{r=1}^{I}\left(S_{r}-z_{r}\right)\right]$.

Transition type 3-b: initial state: $\mathbf{x}=(\mathbf{z}, j, k)$ with $(j, k) \in\{1, \ldots, J\} \times\{1, \ldots, K\}$, action: $i$ with $i \in\{1, \ldots, I\}$ and $z_{i}>0$, next event: part request from customer region $n$ and customer class $p$, next state: $\mathbf{y}=\left(\mathbf{z}-\mathbf{e}_{i}, n, p\right)$. The transition rate is $\lambda_{n p}$ and the transition probability $p_{\mathbf{x}, \mathbf{y}}(i)$ is equal to $\lambda_{n p} /\left[\sum_{t=1}^{J} \sum_{u=1}^{K} \lambda_{t u}+\mu+\right.$ $\left.\mu \sum_{r=1}^{I}\left(S_{r}-r_{m}\right)\right]$. 
Transition type 4-a: initial state: $\mathbf{x}=(\mathbf{z}, j, k)$ with $(j, k) \in\{1, \ldots, J\} \times\{1, \ldots, K\}$, action: 0 , next event: order arrival at local warehouse $m$, next state: $\mathbf{y}=\left(\mathbf{z}+\mathbf{e}_{m}, 0,0\right)$. The transition rate is $\mu\left(S_{m}-z_{m}\right)$ and the transition probability $p_{\mathbf{x}, \mathbf{y}}(0)$ is $\mu\left(S_{m}-z_{m}\right) /\left[\sum_{t=1}^{J} \sum_{u=1}^{K} \lambda_{t u}+\mu \sum_{r=1}^{I}\left(S_{r}-z_{r}\right)\right]$.

Transition type 4-b: initial state: $\mathbf{x}=(\mathbf{z}, j, k)$ with $(j, k) \in\{1, \ldots, J\} \times\{1, \ldots, K\}$, action: $i$ with $i \in\{1, \ldots, I\}$ and $z_{i}>0$, next event: order arrival at local warehouse $m$, next state: $\mathbf{y}=\left(\mathbf{z}-\mathbf{e}_{i}+\mathbf{e}_{m}, 0,0\right)$. If $m \neq i$, the transition rate is $\mu\left(S_{m}-z_{m}\right)$ and the transition probability $p_{\mathbf{x}, \mathbf{y}}(i)$ is $\mu\left(S_{m}-z_{m}\right) /\left[\sum_{t=1}^{J} \sum_{u=1}^{K} \lambda_{t u}+\right.$ $\left.\mu+\mu \sum_{r=1}^{I}\left(S_{r}-z_{r}\right)\right]$. If $m=i$, the transition rate is $\mu\left(S_{i}+1-z_{i}\right)$ and the transition probability is $\mu\left(S_{i}+1-z_{i}\right) /\left[\sum_{t=1}^{J} \sum_{u=1}^{K} \lambda_{t u}+\mu+\mu \sum_{r=1}^{I}\left(S_{r}-z_{r}\right)\right]$.

The mean transition period lengths $\tau(\mathbf{x}, a)$ for all state/action pairs directly follow from the transition rates. For state $(\mathbf{z}, 0,0)$ and action -1 the transition period length $\tau((\mathbf{z}, 0,0),-1)$ is $1 /\left[\sum_{t=1}^{J} \sum_{u=1}^{K} \lambda_{t u}+\right.$ $\left.\mu \sum_{r=1}^{I}\left(S_{r}-z_{r}\right)\right]$. For state $(\mathbf{z}, j, k)$ with $(j, k) \in\{1, \ldots, J\} \times\{1, \ldots, K\}$ and action 0 the transition period length $\tau((\mathbf{z}, j, k), 0)$ is equal to $\tau((\mathbf{z}, 0,0),-1)$. Finally, for state $(\mathbf{z}, j, k)$ with $(j, k) \in\{1, \ldots, J\} \times\{1, \ldots, K\}$ and action $i$ with $i \in\{1, \ldots, I\}$ the transition period length $\tau((\mathbf{z}, j, k), i)$ is $1 /\left[\sum_{t=1}^{J} \sum_{u=1}^{K} \lambda_{t u}+\mu+\right.$ $\left.\mu \sum_{r=1}^{I}\left(S_{r}-z_{r}\right)\right]$. We conclude our MDP formulation with the specification of the expected direct cost $G((\mathbf{z}, j, k), a)$ when choosing action $a$ in state $(\mathbf{z}, j, k)$ :

$$
G((\mathbf{z}, j, k), a)= \begin{cases}C_{a j k}^{f} & \text { if } a \geq 0 \\ 0 & \text { otherwise }\end{cases}
$$

To obtain the optimal allocation rule we transform the continuous-time MDP into a discrete-time MDP by applying a technique called uniformization (see e.g Bertsekas (2007), p288 - 295). The uniformization procedure consists of two steps: In the first step, we determine a new transition period length $\tau$ such that $\tau \leq \tau(\mathbf{x}, a)$ for all $\mathbf{x} \in \mathcal{S}, a \in \mathcal{A}$. It is easy to see that $\tau=1 /\left[\sum_{t=1}^{J} \sum_{u=1}^{K} \lambda_{t u}+\mu \sum_{r=1}^{I} S_{r}\right]$ is an appropriate choice. In the second step, we add two types of transitions: (i) from state $(\mathbf{z}, 0,0)$ and action -1 to $(\mathbf{z}, 0,0)$, and (ii) from state $(\mathbf{z}, j, k)$ and action $i \in \mathcal{A}(\mathbf{z}, j, k)$ to $\left(\mathbf{z}-\mathbf{e}_{i}, 0,0\right)$. The transition rates for these fictitious transitions are chosen such that $\tau(\mathbf{x}, a)=\tau$ for all $\mathbf{x} \in \mathcal{S}$, and $a \in \mathcal{A}(\mathbf{x})$. We obtain the desired discrete-time MDP by replacing the exponentially distributed transition period lengths with constant transition period lengths with the same mean. The Bellman optimality equations for the (discrete-time) average cost MDP now read as:

$$
h^{\star}(\mathbf{x})=\min _{a \in \mathcal{H}(\mathbf{x})}\left[G(\mathbf{x}, a)-\lambda^{\star} \tau+\sum_{\mathbf{y} \in \mathcal{S}} p_{\mathbf{x}, \mathbf{y}}(a) h^{\star}(\mathbf{y})\right] \forall \mathbf{x} \in \mathcal{S}
$$

In this system of equations, $\mathbf{h}^{\star}$ is the optimal relative cost vector, $G(\mathbf{x}, a)$ is the expected direct cost function as defined in (1) and $\lambda^{\star}$ represents the optimal expected cost per time unit. We solve the Bellman optimality equations using relative value iteration (see e.g. Bertsekas (2007), p204 - 229). The optimal action $a^{\star}(\mathbf{x})$ is the action that attains the minimum in (2). 
It is easy to see that if $\left(\mathbf{h}^{\star}, \lambda^{\star}\right)$ is a solution to (2), then $\left(\mathbf{h}^{\star}+b \mathbf{1}, \lambda^{\star}\right)$ with $b \in \mathbb{R}$ is also a solution. This means that one may add an equation of the form $\mathbf{c}^{\mathbf{T}} \mathbf{h}^{\star}=b$ to (2) to make the solution unique. An interesting option is to add the equation with $\mathbf{c}^{\mathbf{T}}$ equal to the state probabilities and $b$ equal to 0 . With this additional equation, the value of $h^{\star}(\mathbf{x})$ can be interpreted as the total additional cost over an infinite time horizon when starting in state $\mathbf{x}$ compared to paying $\lambda^{\star}$ every time unit. This interpretation of $h^{\star}(\mathbf{x})$ plays an important role in the development of our dynamic allocation rule in the next section.

\section{Heuristic allocation rules}

In this section, we present three heuristic allocation rules for the spare parts inventory network discussed in the previous section. The first heuristic allocation rule is a simple static allocation rule (denoted as SArule) which is described in Subsection 4.1. Because this rule is widely used in real life, it constitutes an important benchmark for any newly developed allocation rule. Next, we move to dynamic allocation rules. In this paper, we restrict ourselves to one-step lookahead (1SL) policies. One-step lookahead policies choose at each state $\mathbf{x}$ the action $a^{1 S L}(\mathbf{x})$ that minimizes the sum of the direct cost and an approximation of the optimal future cost:

$$
a^{1 S L}(\mathbf{x})=\arg \min _{a \in \mathcal{A}(\mathbf{x})}\left[G(\mathbf{x}, a)+\sum_{\mathbf{y} \in \mathcal{S}} p_{\mathbf{x}, \mathbf{y}}(a) \hat{h}(\mathbf{y})\right] \quad \forall \mathbf{x} \in \mathcal{S}
$$

If $\hat{\mathbf{h}}$ is the relative cost vector of some heuristic allocation rule (called the base policy), then the one-step lookahead policy is called a rollout policy. For rollout policies, we can simplify (3) considerably. Using the Bellman equations for states $\left(\mathbf{z}-\mathbf{e}_{a}, 0,0\right)$ and action -1 , the transition probabilities for states $\left(\mathbf{z}-\mathbf{e}_{a}, 0,0\right)$ and action -1 , and the transition probabilities for states $(\mathbf{z}, j, k)$ and action $a$, we obtain following intuitive expression:

$$
a^{1 S L}(\mathbf{z}, j, k)=\arg \min _{a \in \mathcal{A}(\mathbf{z}, j, k)}\left[C_{a j k}^{f}+\hat{h}\left(\mathbf{z}-\mathbf{e}_{a}, 0,0\right)\right] \quad \forall(\mathbf{z}, j, k) \in \mathcal{S} \text { and } j>0
$$

The second heuristic allocation rule we present in this section is a rollout allocation policy with the SA-rule as base policy. This policy is denoted as the RA-rule and is described in Subsection 4.2. To derive the RA-rule, we must solve a system of $(J+1) \prod_{i=1}^{I}\left(S_{i}+1\right)$ Bellman equations in order to obtain $\mathbf{h}^{\mathbf{S A}}$, the relative cost vector under the SA-rule. For problem instances of real-life size this is infeasible. To overcome this problem, we develop a one-step lookahead allocation rule where we approximate the optimal relative cost with an estimate of the relative cost under the SA-rule. This third allocation rule is a dynamic allocation rule. It is denoted as the DA-rule and described in Subsection 4.3. 


\subsection{SA-rule}

The SA-rule is a static allocation rule that uses predefined priorities to determine, among all warehouses with stock at hand, the warehouse that is selected to fulfill a part request. It takes as input $J \times K$ sorted lists of warehouses, one for each pair of customer region and customer class. Let $L_{j k}(q)$ denote the $q^{\text {th }}$ warehouse in the static priority list of customer region $j$ and customer class $k$. In case of a part failure at a customer of class $k$ in customer region $j$, a new spare part is sent from the first warehouse in $L_{j k}$ with stock at hand. The warehouses in $L_{j k}$ are sorted in ascending order on the basis of the fulfillment $\operatorname{cost} C_{i j k}^{f}$.

\subsection{RA-rule}

The RA-rule is a rollout policy that uses the SA-rule as base policy. An interesting property of rollout policies is that they have the cost improvement property which states that they achieve no worse results than their base policies. A major disadvantage is that the RA-rule requires calculating $\mathbf{h}^{\mathbf{S A}}$, and this involves solving a (possibly huge) set of Bellman equations. The practical value of the RA-rule is therefore limited. Yet, we have added the RA-rule to our analysis because it is the inspiration for our DA-rule.

\subsection{DA-rule}

In this subsection we present the DA-rule. The DA-rule is a one-step lookahead policy. In contrast to the optimal allocation rule and the RA-rule, it is applicable to problems of arbitrary size. First, we explain the general idea behind the DA-rule. Then, we discuss the three components that together make up the algorithm for approximating the optimal relative cost. We conclude with a formal description of the DA-rule.

\subsubsection{Idea behind the DA-rule}

The DA-rule is a one-step lookahead policy where we approximate the optimal relative cost with an estimate of the relative cost under the SA-rule. The estimate is obtained through a computationally inexpensive iterative procedure. Consequently, the DA-rule can be considered as an approximation of the RA-rule (but one that can handle problems of arbitrary size).

Consider the system when a demand arrives. Without loss of generality we assume that time is 0 and the actual stock level vector is equal to $\mathbf{z}$. We want to approximate $\mathbf{h}^{\mathbf{S A}}$ for all states $\left(\mathbf{z}-\mathbf{e}_{a}, 0,0\right)$ with $z_{a}>0$. Let us define $J\left(\mathbf{z}-\mathbf{e}_{a}, t_{1}, t_{2}\right)$ as the expected total cost during time interval $\left[t_{1}, t_{2}\right]$ if the system is in state $\left(\mathbf{z}-\mathbf{e}_{a}, 0,0\right)$ at time 0 and the SA-rule is used to fulfill future demands. Then, by definition:

$$
h^{S A}\left(\mathbf{z}-\mathbf{e}_{a}, 0,0\right)=\lim _{t \rightarrow \infty}\left[J\left(\mathbf{z}-\mathbf{e}_{a}, 0, t\right)-\lambda^{S A} t\right]
$$

Our approximation of $\mathbf{h}^{\mathbf{S A}}$ is based on a similar idea as that in Axsäter (1990). We make a decomposition of the network into individual local warehouses. In this way, we only deal with one Markov process per 
local warehouse instead of one Markov process for the entire network. Under the SA-rule, all demand from customer region $j$ and customer class $k$ is first offered to warehouse $L_{j k}(1)$. If demand arrives when $L_{j k}(1)$ has no stock at hand, it is offered to $L_{j k}(2)$. If $L_{j k}(2)$ is also out of stock, it is offered to $L_{j k}(3)$, et cetera. We assume that the overflow demand stream from customer region $j$ and customer class $k$ to warehouse $i \neq L_{j k}(1)$ is a Poisson process. Consequently, each warehouse can be evaluated individually as an Erlang loss model $\left(M|M| S_{i} \mid S_{i}\right.$ queue). In earlier contributions, Axsäter (1990), Alfredsson \& Verrijdt (1999), Kukreja et al. (2001), Kutanoglu (2008), Kranenburg \& Van Houtum (2009), and Reijnen et al. (2009) have derived approximations for the average network flow rates in steady state. However, since we want to calculate the relative (sometimes called differential) cost, we are interested in transient system behavior. To the best of our knowledge this has not been looked at before.

We approximate the transient system behavior in the following way: We assume for each warehouse $i$ a (constant) stockout probability $p_{i}$ during $[0, T]$ and the steady state stockout probability $\bar{p}_{i}$ when $t>T$. The parameter $T \geq 0$ is a design parameter for the DA-rule. As default we use $T=\frac{1}{\mu}$. Later in this subsection we derive an expression for $p_{i}$. At this point, we just mention that it depends on the initial stock level vector $\left(\mathbf{z}-\mathbf{e}_{a}\right)$. Next, we assume stationary demand streams during $[0, T]$ that follow from the stockout probabilities $p_{i}$, and stationary demand streams when $t>T$ that follow from the stockout probabilities $\bar{p}_{i}$. From the estimated stationary demand rates and the estimated stationary stockout probabilities we can immediately estimate the stationary flow rates in the network for $[0, T]$ and $t>T$. We can use these estimated stationary flow rates to derive approximations for $J\left(\mathbf{z}-\mathbf{e}_{a}, 0, T\right)$ and $J\left(\mathbf{z}-\mathbf{e}_{a}, T, t\right)$ for arbitrary $t>T$. For the purpose of our analysis, we now rewrite (5) as:

$$
h^{S A}\left(\mathbf{z}-\mathbf{e}_{a}, 0,0\right)=J\left(\mathbf{z}-\mathbf{e}_{a}, 0, T\right)+\lim _{t \rightarrow \infty}\left[J\left(\mathbf{z}-\mathbf{e}_{a}, T, t\right)-\lambda^{S A} t\right]
$$

Let $\hat{J}()$ denote our approximation of $J()$, and let $\hat{h}^{S A}\left(\mathbf{z}-\mathbf{e}_{a}\right)$ denote our approximation of $h^{S A}\left(\mathbf{z}-\mathbf{e}_{a}\right)$. Then, using the assumption that the system is in steady state for $t>T$ and removing all parts that do not depend on $\left(\mathbf{z}-\mathbf{e}_{a}, 0,0\right)$, we get $\hat{h}^{S A}\left(\mathbf{z}-\mathbf{e}_{a}, 0,0\right)=\hat{J}\left(\mathbf{z}-\mathbf{e}_{a}, 0, T\right)$. Plugging this into (4), we obtain the following expression for the DA-rule:

$$
a^{D A}(\mathbf{z}, j, k)=\arg \min _{a \in \mathcal{A}(\mathbf{z}, j, k)}\left[C_{a j k}^{f}+\hat{J}\left(\mathbf{z}-\mathbf{e}_{a}, 0, T\right)\right] \quad \forall(\mathbf{z}, j, k) \in \mathcal{S} \text { and } j>0
$$

The algorithm for calculating $\hat{J}\left(\mathbf{z}-\mathbf{e}_{a}, 0, T\right)$ consists of three main steps. The first two steps are executed alternately and provide an accurate estimate of the average network flow during $[0, T]$ when starting at the actual stock level vector $\left(\mathbf{z}-\mathbf{e}_{a}\right)$ at time 0 . Before we describe these two steps in detail, we introduce some notation: Let $D_{i j k}$ denote (an approximation of) the average demand rate of customer class $k$ in customer region $j$ to warehouse $i$ during $[0, T]$ and let $p_{i}$ denote (an approximation of) the average stockout probability at warehouse $i$ during $[0, T]$. 
Step 1: Calculate the stockout probabilities $p_{i}$ given the demand rates $D_{i j k}$, and the initial stock level vector $\left(\mathbf{z}-\mathbf{e}_{a}\right)$.

Step 2: Update the demand rates $D_{i j k}$ given the average stockout probabilities $p_{i}$.

The two steps are executed until the changes in $D_{i j k}$ in two consecutive iterations are smaller than some pre-specified, small value $\epsilon$. The iterative process is initialized with $D_{i j k}=\lambda_{j k}$ if $i=L_{j k}(1)$ and $D_{i j k}=0$ otherwise. In the third step, we calculate our approximation of the relative cost under the SA-rule for state $\left(\mathbf{z}-\mathbf{e}_{a}, 0,0\right)$.

Step 3: Calculate $\hat{J}\left(\mathbf{z}-\mathbf{e}_{a}, 0, T\right)$, given the converged values of $D_{i j k}$ and $p_{i}$.

We now explain these three steps in more detail.

\subsubsection{Step 1: Calculating $p_{i}$ for given demand rates $D_{i j k}$}

Because we have assumed that the overflow demand streams are Poisson, we can model each warehouse $i$ as an Erlang loss model with $S_{i}$ servers, demand arrival rate $D_{i}=\sum_{j} \sum_{k} D_{i j k}$ and service rate $\mu$. Suppose that the initial number of free servers (in our setting this is equivalent to the actual stock level) is equal to $z_{i}$. We are interested in approximating the average stockout probability during time interval $[0, T]$. The steady state stockout probability in an Erlang loss model can be calculated from the well-known Erlang B formula:

$$
B\left(S_{i}, \frac{D_{i}}{\mu}\right)=\frac{\frac{1}{S_{i} !}\left(\frac{D_{i}}{\mu}\right)^{S_{i}}}{\sum_{x=0}^{S_{i}} \frac{1}{x !}\left(\frac{\lambda}{\mu}\right)^{x}}
$$

Let $N\left(S_{i}, D_{i}, \mu, z_{i}, t\right)$ denote the expected number of rejected requests in the $M|M| S_{i} \mid S_{i}$ queue during time interval $[0, t]$ starting with $z_{i}$ items in stock at time 0 . Furthermore, let $\Delta\left(S_{i}, D_{i}, \mu, z_{i}\right)$ represent the additional number of rejected requests over an infinite time horizon starting with $z_{i}$ items in stock at time 0 , compared to steady state. The formal definition is given below:

$$
\Delta\left(S_{i}, D_{i}, \mu, z_{i}\right)=\lim _{t \rightarrow \infty}\left[N\left(S_{i}, D_{i}, \mu, z_{i}, t\right)-D_{i} t B\left(S_{i}, \frac{D_{i}}{\mu}\right)\right]
$$

Our idea is to approximate the transient stockout probability with a step-function. For $t \leq T$ we use a constant stockout probability $p_{i}$, and for $t>T$ we use the steady state stockout probability $\bar{p}_{i}=B\left(S_{i}, \frac{D_{i}}{\mu}\right)$. To calculate $p_{i}$ we assume that the additional number of rejected requests compared to steady state all occur in the time interval $[0, T]$. Under this assumption the expected number of rejected requests during $[0, T]$ is equal to $D_{i} T B\left(S_{i}, \frac{D_{i}}{\mu}\right)+\Delta\left(S_{i}, D_{i}, \mu, z_{i}\right)$. Consequently, we can approximate the average stockouts probability during $[0 T]$ as follows:

$$
p_{i}=F\left(S_{i}, D_{i}, \mu, z_{i}, T\right)=\max \left\{0, \min \left\{1, B\left(S_{i}, \frac{D_{i}}{\mu}\right)+\frac{\Delta\left(S_{i}, D_{i}, \mu, z_{i}\right)}{D_{i} T}\right\}\right\}
$$


The min and max in expression (10) have been added to make sure that the calculated stockout probabilities are never smaller than zero or bigger than one. Before we can apply (10), we must calculate $\Delta\left(S_{i}, D_{i}, \mu, z_{i}\right)$.

To obtain $\Delta\left(S_{i}, D_{i}, \mu, z_{i}\right)$, the following steps are carried out: (i) we formulate the $M|M| S_{i} \mid S_{i}$ queue that represents warehouse $i$ as an average cost MDP with a cost of 1 in case of a rejected customer demand, (ii) we construct a set of equations for the relative cost vector $\mathbf{h}=\left(h_{0}, h_{1}, \ldots, h_{S_{i}}\right)$ consisting of $\left(S_{i}+1\right)$ Bellman equations and one scaling equation, and (iii) we solve this system of equations. The scaling constraint is chosen such that $\Delta\left(S_{i}, D_{i}, \mu, z_{i}\right)=h_{z_{i}}$. We now explain these steps in more detail.

To formulate the $M|M| S_{i} \mid S_{i}$ queue as an average cost MDP we choose as state the number of items on stock. We have two events: (i) arrival of a customer demand, and (ii) arrival of a replenishment order, and we also have two actions: (i) fulfill demand, and (ii) reject demand. For every state we have exactly one admissible action (defined by the nature of the $M|M| S_{i} \mid S_{i}$ queue): Demand is fulfilled if there is stock at hand, and rejected if there is no stock at hand. We incur unit cost for each rejected demand. The Bellman equations for this system read as:

$$
\begin{array}{lllll}
h_{0}=\frac{D_{i}}{D_{i}+S_{i} \mu}-\frac{D_{i} B\left(S_{i}, \frac{D_{i}}{\mu}\right)}{D_{i}+S_{i} \mu}+\frac{D_{i}}{D_{i}+S_{i} \mu} & h_{0}+\frac{S_{i} \mu}{D_{i}+S_{i} \mu} & h_{1} \\
h_{n}= & -\frac{D_{i} B\left(S_{i}, \frac{D_{i}}{\mu}\right)}{D_{i}+\left(S_{i}-n\right) \mu}+\frac{D_{i}}{D_{i}+\left(S_{i}-n\right) \mu} & h_{n-1}+\frac{\left(S_{i}-n\right) \mu}{\lambda+\left(S_{i}-n\right) \mu} & h_{n+1} \\
& & & 1 \leq n \leq S_{i}-1 \\
h_{S_{i}}= & -\frac{D_{i} B\left(S_{i}, \frac{D_{i}}{\mu}\right)}{D_{i}}+ & h_{S_{i}-1} &
\end{array}
$$

Next, we add a scaling equation to (11) such that the resulting system of equations has a unique solution with $\Delta\left(S_{i}, D_{i}, \mu, z_{i}\right)=h_{z_{i}}$. This constraint states that weighted sum of all vector elements of $\mathbf{h}$ must be equal to zero where the weights are equal to the state probabilities. It reads as:

$$
\sum_{n=0}^{S_{i}}\left[\frac{\frac{1}{n !}\left(\frac{D_{i}}{\mu}\right)^{n}}{\sum_{x=0}^{S_{i}} \frac{1}{x !}\left(\frac{D_{i}}{\mu}\right)^{x}}\right] h_{n}=0
$$

We obtain $h_{z_{i}}$ (and thus $\left.\Delta\left(S_{i}, D_{i}, \mu, z_{i}\right)\right)$ by solving the system of equations consisting of (11) and (12). The simple structure of the system of equations allows for a fast sequential solution procedure. We have illustrated our step-function approximation of the real transient stockout probability for an arbitrary local warehouse in Example 1.

Example 1. Consider a local warehouse $i$ with base stock level $S_{i}=3$ and suppose that the average replenishment lead time $\frac{1}{\mu}$ is equal to $\frac{1}{5}$. Furthermore, suppose that at a certain point in the iterative procedure for calculating $\hat{J}\left(\mathbf{z}-\mathbf{e}_{a}, 0, T\right)$, the demand arrival rate $D_{i}=\sum_{j} \sum_{k} D_{i j k}$ for warehouse $i$ is equal 
to 12. Warehouse $i$ can now be modeled as an $M|M| 3 \mid 3$ queue with an average service time of $\frac{1}{5}$, and a demand arrival rate of 12. To investigate how the stockout probability at warehouse i evolves over time, we have written a Matlab simulation script. Figure 1(a) shows the real stockout probability as a function of time for 0 items in stock at time 0 and Figure 1(b) show our approximation of the real stockout probability with $T=\frac{1}{\mu}$. Note that the shaded areas in Figures $1(a)$ and $1(b)$ are equal.

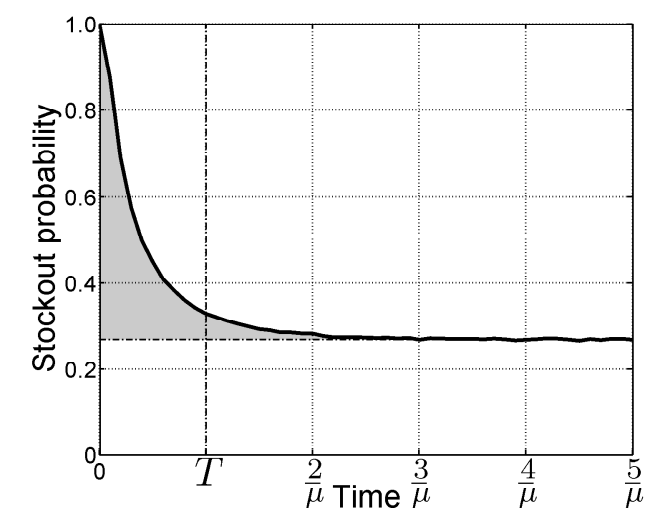

(a) Real transient behavior for $z=0$

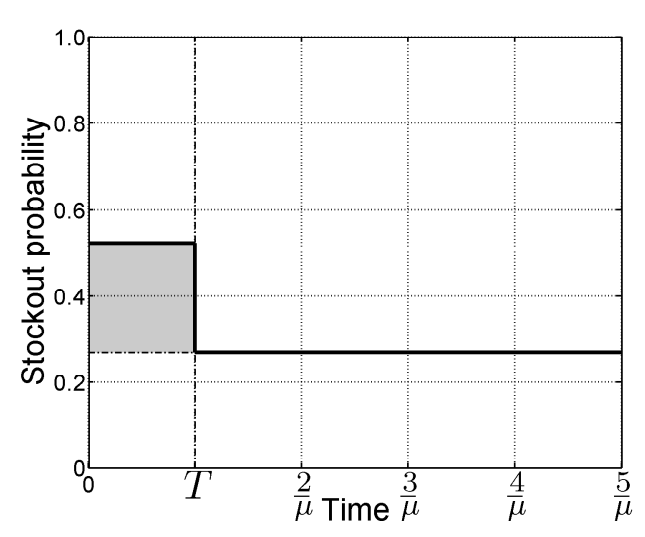

(b) Step-function approximation for $z=0$

Figure 1: Real and approximated transient stockout probability for $M|M| 3 \mid 3$ queue

\subsubsection{Step 2: Updating $D_{i j k}$ for given average stockout probabilities $p_{i}$}

By definition of the SA-rule, all demand from customer region $j$ and customer class $k$ is offered to $L_{j k}(1)$. Suppose that warehouse $i=L_{j k}(1)$ is out of stock a fraction $p_{i}$ of the time. Then, a fraction $\left(1-p_{i}\right)$ of the demand from customer region $j$ and customer class $k$ is fulfilled from warehouse $i$, and an overflow demand stream $p_{i} \lambda_{j k}$ is offered to warehouse $L_{j k}(2)$. In general, the overflow demand rates $D_{i j k}$ can be recursively determined from $D_{L_{j k}(q), j, k}=p_{L_{j k}(q-1)} D_{L_{j k}(q-1), j, k}$. Hence,

$$
D_{L_{j k}(q), j, k}=\left\{\begin{array}{lll}
\lambda_{j k} & \text { if } & q=1 \\
\lambda_{j k} \prod_{n=1}^{q-1} p_{L_{j k}(q)} & \text { if } & q>1
\end{array}\right.
$$

Note that in this recursive formula we use the simplifying assumption that actual stock levels at the local warehouses are independent.

\subsubsection{Step 3: Calculating $\hat{J}\left(\mathbf{z}-\mathbf{e}_{a}, 0, T\right)$ for given $D_{i j k}$ and $p_{i}$.}

We can estimate the average flow rate of spare parts for customer class $k$ during $[0, T]$ between warehouse $i$ and customer region $j$ by multiplying the (estimated) average demand rate during $[0, T]$ with the (estimated) average fill rate of warehouse $i$ during $[0, T]$. We obtain an estimate of the average total cost during $[0, T]$ by taking the sum over all network edges of the product of the estimated flow rate (i.e. $\left.\left(1-p_{i}\right) D_{i j k}\right)$, the fulfillment cost (i.e. $C_{i j k}^{f}$ ), and the length of the time horizon (i.e. $T$ ). 


\subsubsection{Formal specification}

We conclude this subsection with a formal description of the DA-rule. It consists of the control rule (7) and the Algorithm 1 for calculating $\hat{J}\left(\mathbf{z}-\mathbf{e}_{a}, 0, T\right)$. To evaluate the performance on problem instances of real-life size, we have implemented the DA-rule in a JAVA simulation program. Each time the simulator generates a new part request, we execute Algorithm 1 for all warehouses with stock at hand. No allocation decisions or relative cost vectors are stored. Consequently, storage space requirements are minimal. The average computation time per part request is less than 10 milliseconds on an Intel T2600 $2 \mathrm{GHz}$ computer for all problem instances in our numerical experiments (including the ones of real-life size).

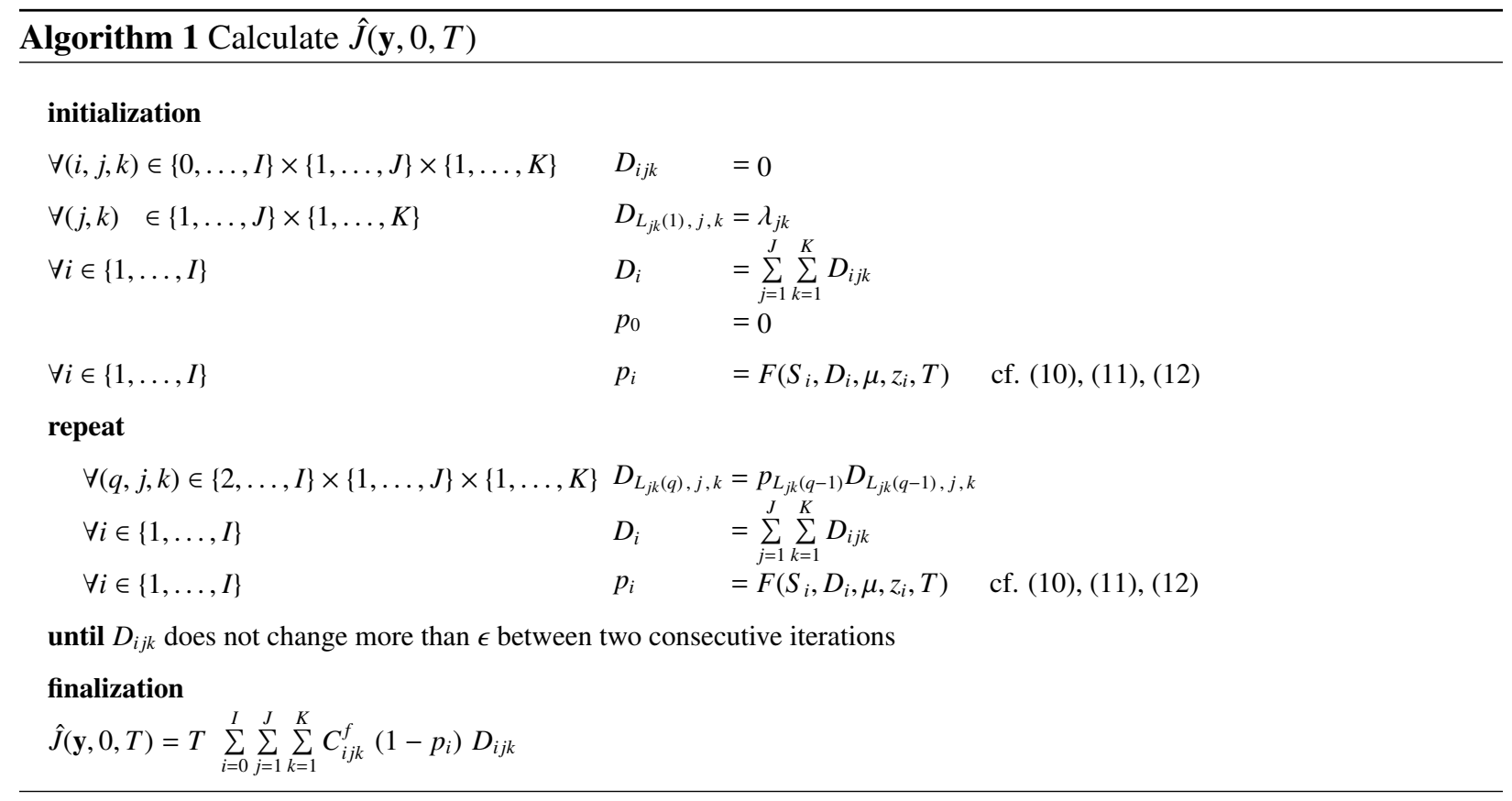

\section{Numerical Experiments}

In this section, we investigate the performance and the structure of the proposed DA-rule via numerical experiments. We have the following objectives in conducting numerical experiments. First, we investigate the optimality gap of the DA-rule. Second, we investigate the impact of the two main approximation steps in the DA-rule: (i) using one-step lookahead with the SA-rule as base policy, and (ii) approximating the true relative cost under the SA-rule with $\hat{J}\left(\mathbf{z}-\mathbf{e}_{i}, 0, T\right)$. Our third objective is to characterize the DA-rule by investigating how it differs from the SA-rule. Finally, we investigate the potential cost savings of the DArule over the SA-rule on problem instances of real-life size, and we explore how the relative performance depends on various problem characteristics.

To meet the first three objectives we define a numerical experiment with a test bed containing a wide range of problem instances of small size (Experiment I). For all problem instances in this test bed, we can 
calculate the computationally expensive optimal allocation rule and the computationally expensive RA-rule. In this experiment we use relative value iteration to evaluate the performance of the allocation rules. To meet the fourth objective we define a numerical experiment with a test bed with problem instances of real-life size (Experiment II) and use discrete event simulation to evaluate the performance of the SA-rule and the DA-rule. In Subsection 5.1 we define the test beds, in Subsection 5.2 we describe the experiment with problem instances of small size, in Subsection 5.3 we characterize the DA-rule, and in Subsection 5.4 we describe the experiment with problem instances of real-life size.

\subsection{Test Bed}

For Experiment I and Experiment II we use similar test beds based on full factorial designs on six parameters. Six more parameters are fixed within each test bed. The main difference between both experiments is that for Experiment I we create test instances of small size (6 local warehouses), whereas for Experiment II we create test instances of real-life size (24 local warehouses). In both experiments, we consider 3 customer classes: customers with 2 hours contracts, customers with 4 hours contracts, and customers with 8 hours contracts. We start by summarizing our choices for all 12 parameter in Table 2. Costs are expressed in euros, times are expressed in hours, and distances are expressed in kilometers.

The total number of all possible combinations for these parameters is $2 \times 3 \times 3 \times 3 \times 3 \times 3=486$ for Experiment I, and $2 \times 3 \times 3 \times 3 \times 3 \times 4=648$ instances for Experiment II. We have randomly created 5 different sets of values for the coordinates of the customer regions, as there are uniform distributions involved in the generation of these values. This gives us in total 486 × $5=2430$ instances for Experiment I and $648 \times 5=3240$ instances for Experiment II.

We now explain some of the parameters in more detail and describe how to construct test instances from the parameters values. The first parameter in the factorial design is the region indicator $(R)$. It can take two values $r_{1}$ or $r_{2}$. The value $r_{1}$ represents a situation where the service area is at relatively close distance to the central warehouse; we use a small average replenishment lead time (72 hours) and a small emergency delivery time (4 hours). The value $r_{2}$ represents a situation where the service area is at relatively large distance from the central warehouse; we use a larger average replenishment lead time (120 hours) and a larger emergency delivery time (8 hours).

The second parameter in the factorial design is the length of a service area unit $(l)$, which determines the network layout. For all test instances, we create a rectangular service area consisting of $3 \times 2$ (Experiment I) or $6 \times 4$ (Experiment II) squares of $l \times l$ kilometers each. We position a local warehouse in the center of each square. For both experiments, the largest value of $l$ is chosen such that an arbitrary part request at an arbitrary point inside the service area can in principle be fulfilled within the service deadline. Since we assumed $t_{i j}=0.5+0.01 d_{i j}$, we can travel 150 kilometers within 2 hours (= minimum service deadline). 


\begin{tabular}{|c|c|c|}
\hline Name of parameter & Values Experiment I & Values Experiment II \\
\hline No. of local warehouses $(I)$ & $\{6\}$ & $\{24\}$ \\
\hline No. of customer regions $(J)$ & $\{24\}$ & $\{96\}$ \\
\hline Delivery time profile $(i>0)$ & $t_{i j}=0.5+0.01 d_{i j}$ & as Experiment I \\
\hline Delivery cost profile $(i>0)$ & $C_{i j}^{d}=$ & as Experiment I \\
\hline Emergency delivery $\operatorname{cost}\left(C_{0 j}^{d}\right)$ & $\{2000\}$ & as Experiment I \\
\hline Replenishment cost $\left(C_{i}^{r}\right)$ & $\{0\}$ & as Experiment I \\
\hline Region indicator $(R)$ & $\left\{r_{1}, r_{2}\right\}$ & as Experiment I \\
\hline \multicolumn{3}{|l|}{$r_{1}: \frac{1}{\mu}=72, t_{0 j}=4$} \\
\hline \multicolumn{3}{|l|}{$r_{2}: \frac{1}{\mu}=120, t_{0 j}=8$} \\
\hline Length service area unit $(l)$ & $\left\{\sqrt{2} \cdot 150,150, \frac{1}{3} \sqrt{6} \cdot 150\right\}$ & as Experiment I \\
\hline Customer class fractions (w) & $\left\{\left(\frac{1}{6}, \frac{2}{6}, \frac{3}{6}\right),\left(\frac{2}{6}, \frac{2}{6}, \frac{2}{6}\right),\left(\frac{3}{6}, \frac{2}{6}, \frac{1}{6}\right)\right\}$ & as Experiment I \\
\hline Relative network demand $(\phi)$ & $\{0.2,0.5,1.0\}$ & as Experiment I \\
\hline Penalty cost vector $\left(\mathbf{C}^{\mathbf{p}}\right)$ & $\{1,2,4\} \times(1200,600,300)$ & as Experiment I \\
\hline Target time-based fill rate $(\boldsymbol{\Gamma})$ & $\{0.5,0.8,0.95\} \times \mathbf{1}$ & $\{0.5,0.8,0.95,0.98\} \times \mathbf{1}$ \\
\hline
\end{tabular}

Table 2: Parameter choices for the test beds

Consequently, the largest value for $l$ in the factorial design is equal to $150 \sqrt{2}$. For all test instances we create $J=4 \cdot I$ customer regions. Each customer region has coordinates that determine the delivery times and the delivery costs. Each customer region generates 2 hours, 4 hours, and 8 hours spare part demands. The location of each customer region is drawn according to a uniform distribution.

The third parameter in the factorial design is the weight vector $\mathbf{w}=\left(w_{1}, w_{2}, w_{3}\right)$ of the customer classes. It specifies for each customer region the fraction of demand associated with a service deadline of 2 hours, 4 hours, and 8 hours, respectively. In our experiments, we do not only assume that all customer regions have identical customer class weights, but also that their total demand rates are identical. A graphical illustration

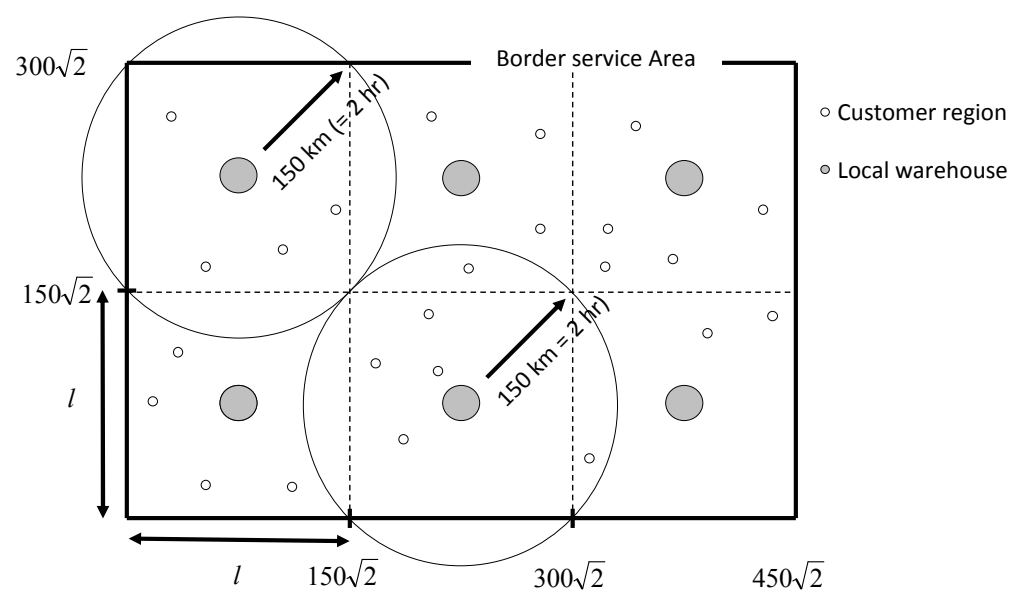

Figure 2: Example of network layout in Experiment I 
of a possible network layout for a problem instance in Experiment I with $l=150 \sqrt{2}$ is given in Figure 2.

The fourth parameter in the factorial design is the average network demand during the replenishment lead time divided by the number of local warehouses. We refer to this parameter as the relative network demand $(\phi)$. From the factorial design parameters $\phi$ and $\mathbf{w}$ we obtain the problem inputs $\lambda_{j k}$ according to:

$$
\lambda_{j k}=\frac{w_{k}}{J} \phi I \mu
$$

The fifth parameter in the factorial design is the hourly penalty cost vector $\mathbf{C}^{\mathbf{p}}$. For each customer class it specifies the penalty cost incurred per hour that a part request is fulfilled beyond the service deadline. Typically, the hourly penalty costs get bigger when the contractual maximum response times get smaller (so $C_{1}^{p}>C_{2}^{p}>C_{3}^{p}$ ).

The sixth and last parameter in the factorial design is the $K$-dimensional vector $\boldsymbol{\Gamma}$ which contains a target time-based fill rate for each customer class. We use $\boldsymbol{\Gamma}$ to calculate the base stock level vector $\mathbf{S}$. To do so, we develop a heuristic that is similar to the one presented in Reijnen et al. (2009). Here, we summarize our heuristic for calculating the base stock levels from the target time-based fill rates. In the first step, we set all base stock levels equal to zero. In the second step, we execute an iterative procedure where we increase the base stock level that minimizes the sum of delivery cost and penalty cost until the time-based fill rates that follow from the network flow approximation of Reijnen et al. (2009) are bigger than the target time-based fill rates. A formal description of the method is given in Appendix A.

\subsection{Experiment $I$}

In this experiment we investigate the optimality gaps of the SA-rule, the RA-rule, and the DA-rule on the test bed defined in the previous subsection. For each of the 2430 test instances we have calculated the total cost, the regular delivery cost, the emergency delivery cost, and the penalty cost for all allocation rules by solving the balance equations for the Markov chain induced by the allocation rule. Consequently, all cost figures presented in this experiment are exact.

We start by providing insight into the spread of the optimality gaps over the 2430 test instances for the three heuristic allocation rules. For this purpose, we calculate for each allocation rule the 5\%, 20\%, 50\%, $80 \%$, and $95 \%$ percentile optimality gap, as well as the minimum and the maximum optimality gap. The results are shown in Table 3. The table shows that the median optimality gap of the SA-rule is $4.4 \%$. For $20 \%$ of all test instances the optimality gap is more than $15 \%$, and the maximum optimality gap is even more than $85 \%$. So, in a considerable part of all test instances there is big room for improvement. The numbers for the RA-rule look totally different. We see that for most test instances the optimality gap of the RA-rule is less than one percent although the maximum optimalty gap of the RA-rule is considerable (18.2\%). For the DA-rule, the $5 \%, 20 \%, 50 \%, 80 \%$, and $95 \%$ optimality gap percentiles are a little bit higher than those of 
RA-rule, but still pretty small. A remarkable observation is that the maximum optimality gap of the DA-rule is smaller than the maximum optimality gap of the RA-rule. So apparantly, the DA-rule can outperform the RA-rule for individual test instances.

\begin{tabular}{lccc}
\hline Percentile & \multicolumn{3}{c}{ Optimality gap (\%) } \\
& SA-rule & RA-rule & DA-rule \\
\hline $\min$ & 0.0 & 0.0 & 0.0 \\
$5 \%$ & 0.3 & 0.0 & 0.0 \\
$20 \%$ & 1.0 & 0.0 & 0.2 \\
$50 \%$ & 4.4 & 0.0 & 0.9 \\
$80 \%$ & 15.6 & 0.5 & 2.8 \\
$95 \%$ & 37.0 & 4.3 & 5.9 \\
$\max$ & 85.7 & 18.2 & 12.1 \\
\hline
\end{tabular}

Table 3: Experiment I: Spread of optimality gap

Next, we calculate the average optimality gap for all three heuristic allocation rules over (i) all 2430 test instances, and (ii) all subsets where one of the factorial design parameters takes one of its admissible values. Besides the average optimality gap (shown in bold face), we also show the contribution of the regular delivery cost, the emergency delivery cost, and the penalty cost to the optimality gap (these values are shown in parenthesis). The results are shown in Table 4.

The first row in Table 4 shows that the average optimality gap over all test instances for the SA-rule, RArule, and DA-rule is $9.6 \%, 0.7 \%$, and $1.6 \%$ respectively. The average optimality gap of the RA-rule is thus more than a factor 10 smaller than the average optimality gap of the SA-rule. This does not only hold for the average optimality gap over all test instances, but also for the average optimality gap in 16 out of 17 subsets. From all this, we conclude that the RA-rule consistently performs very well on this test bed. Unfortunately, the RA-rule cannot be applied to problem instances of real-life size due to computational complexity. That is why we have developed the DA-rule. The table also shows that the average optimality gap of the DA-rule is about a factor 2 bigger than the average optimality gap of the RA-rule. So apparently, approximating the true optimal relative cost $h^{\star}\left(\mathbf{z}-\mathbf{e}_{i}, 0,0\right)$ with $h^{S A}\left(\mathbf{z}-\mathbf{e}_{i}, 0,0\right)$, and approximating $h^{S A}\left(\mathbf{z}-\mathbf{e}_{i}, 0,0\right)$ with $\hat{J}\left(\mathbf{z}-\mathbf{e}_{i}, 0, \frac{1}{\mu}\right)$, both account for about half of the observed optimality gap of the DA-rule. When switching from the SArule to the DA-rule, we achieve an average cost reduction of $100 \% \times(109.6-101.6) / 109.6=7.3 \%$. From the decomposition of the optimality gap into the three different cost components, we see that this cost reduction is mainly obtained by replacing regular shipments by emergency shipments (resulting in higher emergency delivery cost but lower penalty cost). In the next subsection, we investigate the differences between the SA-rule and the RA-rule in more detail. Finally, Table 4 also provides valuable information 


\begin{tabular}{|c|c|c|c|c|}
\hline \multirow[t]{2}{*}{ Test bed subset } & \multirow[t]{2}{*}{$\mathbf{N}$} & \multicolumn{3}{|c|}{ Average optimality gap (\%) } \\
\hline & & SA-rule & RA-rule & DA-rule \\
\hline All instances & 2430 & $9.6(-0.7,-5.7,16.0)$ & $\mathbf{0 . 7}(-0.0,2.3,-1.6)$ & $\mathbf{1 . 6}(0.3,-1.6,3.0)$ \\
\hline$R=r_{1}$ & 1215 & $5.8(-1.0,-3.9,10.7)$ & $\mathbf{0 . 5}(0.1,1.4,-1.0)$ & $1.2(0.2,-1.5,2.1)$ \\
\hline$R=r_{2}$ & 1215 & $13.3(-0.5,-7.5,21.3)$ & $\mathbf{0 . 9}(-0.1,3.2,-2.2)$ & $2.1(0.4,-1.8,3.5)$ \\
\hline$l=\sqrt{2} \cdot 150$ & 810 & $\mathbf{8 . 0}(-1.7,-3.6,13.3)$ & $\mathbf{0 . 5}(0.1,1.5,-1.1)$ & $\mathbf{1 . 0}(0.2,-1.3,2.1)$ \\
\hline 150 & 810 & $9.5(-0.6,-5.8,15.0)$ & $\mathbf{0 . 8}(-0.0,2.5,-1.7)$ & $\mathbf{1 . 8}(0.3,-1.8,3.3)$ \\
\hline$l=\frac{1}{3} \sqrt{6} \cdot 150$ & 810 & $11.2(0.0,-7.6,18.8)$ & $\mathbf{0 . 8}(-0.1,2.9,-2.0)$ & $2.1(0.3,-1.8,3.6)$ \\
\hline $\mathbf{w}=\left(\frac{1}{6}, \frac{2}{6}, \frac{3}{6}\right)$ & 810 & $7.4(-0.1,-5.1,12.6)$ & $\mathbf{0 . 8}(-0.0,2.7,-1.6)$ & $1.4(0.2,-1.2,2.4)$ \\
\hline $\mathbf{w}=\left(\frac{2}{6}, \frac{2}{6}, \frac{2}{6}\right)$ & 810 & $10.8(-0.9,-6.3,18.0)$ & $\mathbf{0 . 8}(-0.2,2.7,-1.9)$ & $\mathbf{1 . 8}(0.3,-1.8,3.3)$ \\
\hline $\mathbf{w}=\left(\frac{3}{6}, \frac{2}{6}, \frac{1}{6}\right)$ & 810 & $10.6(-1.2,-5.6,17.4)$ & $\mathbf{0 . 5}(0.2,1.5,-1.2)$ & $\mathbf{1 . 8}(0.4,-1.9,3.3)$ \\
\hline$\phi=0.2$ & 810 & $3.4(-0.7,-2.8,6.9)$ & $\mathbf{0 . 0}(0.0,0.2,-0.2)$ & $0.5(0.2,-0.3,0.6)$ \\
\hline$\phi=0.5$ & 810 & $9.1(-0.9,-5.8,15.8)$ & $\mathbf{0 . 5}(-0.0,2.0,-1.5)$ & $\mathbf{1 . 6}(0.1,-1.7,3.2)$ \\
\hline$\phi=1.0$ & 810 & $16.1(-0.6,-8.5,25.3)$ & $1.6(-0.1,4.7,-3.1)$ & $2.8(0.5,-2.9,5.1)$ \\
\hline $\mathbf{C}^{\mathbf{p}}=(1200,600,300)$ & 810 & $2.8(0.0,-2.6,5.4)$ & $\mathbf{0 . 1}(0.0,0.4,-0.4)$ & $\mathbf{0 . 9}(0.2,-1.3,2.1)$ \\
\hline $\mathbf{C}^{\mathbf{p}}=(2400,1200,600)$ & 810 & $7.7(-0.6,-5.3,13.6)$ & $\mathbf{0 . 4}(-0.0,1.9,-1.4)$ & $\mathbf{1 . 6}(0.2,-1.7,3.1)$ \\
\hline $\mathbf{C}^{\mathbf{p}}=(4800,2400,1200)$ & 810 & $18.2(-1.6,-9.2,29.0)$ & $1.6(-0.0,4.7,-3.1)$ & $2.4(0.5,-1.8,3.7)$ \\
\hline $\boldsymbol{\Gamma}=(0.50,0.50,0.50)$ & 810 & $\mathbf{1 4 . 5}(0.6,-10.7,24.6)$ & $1.2(-0.4,4.2,-2.6)$ & $1.4(0.3,-1.7,2.8)$ \\
\hline $\boldsymbol{\Gamma}=(0.80,0.80,0.80)$ & 810 & $9.7(-1.4,-5.3,16.4)$ & $\mathbf{0 . 7}(0.1,2.4,-1.7)$ & $2.2(0.3,-2.5,4.4)$ \\
\hline $\boldsymbol{\Gamma}=(0.95,0.95,0.95)$ & 810 & $4.5(-1.4,-1.1,7.0)$ & $\mathbf{0 . 2}(0.3,0.5,-0.5)$ & $\mathbf{1 . 3}(0.3,-0.7,1.7)$ \\
\hline
\end{tabular}

Table 4: Experiment I: optimality gap

on the dependencies between the factorial design parameters and the optimality gaps for three heuristic allocation rules. The strongest dependencies are found for the relative network demand $(\phi)$, the penalty cost vector $\left(\mathbf{C}^{\mathbf{p}}\right)$, and the target time-based fill rate vector $(\boldsymbol{\Gamma})$. For all three heuristic allocation rules, the dependencies point in the same direction, meaning that they all have relatively large optimality gaps for the same kind of test instances. In particular, optimality gaps seem relatively large for problem instances where contract violations occur frequently (low $\mathbf{\Gamma}$ ) and are penalized strongly (big $\mathbf{C}^{\mathbf{p}}$ ), and the probability of multiple demands within one replenishment lead time is relatively high (big $\phi)$.

To investigate the impact of the time horizon $T$ on the performance of the DA-rule, we have compared the DA-rule with $T=\frac{1}{\mu}$ (our default), with the DA-rules with $T=\frac{1}{2} \frac{1}{\mu}, T=2 \cdot \frac{1}{\mu}$, and $T=4 \cdot \frac{1}{\mu}$ on all 2430 instances of Experiment I. The results are summarized in Table 5 and indicate that our default value $T=\frac{1}{\mu}$ is a good choice. Therefore, we use $T=\frac{1}{\mu}$ in the remainder of this paper. 


\begin{tabular}{ccccc}
\hline & $\boldsymbol{T}=\frac{\mathbf{1}}{\mathbf{2}} \frac{\mathbf{1}}{\mu}$ & $\boldsymbol{T}=\frac{\mathbf{1}}{\mu}$ & $\boldsymbol{T = 2} \cdot \frac{\mathbf{1}}{\mu}$ & $\boldsymbol{T = 4} \cdot \frac{\mathbf{1}}{\mu}$ \\
\hline Average optimality gap DA-rule (\%) & 2.4 & 1.6 & 2.8 & 4.1 \\
\hline
\end{tabular}

Table 5: Experiment I: impact of $T$ on performance DA-rule

\subsection{Characterization of DA-rule}

In this subsection we aim to characterize the DA-rule. We do this by identifying the states where the DArule takes a different decision than the (simple) SA-rule. For our analysis we use the test bed of Experiment I. We start by decomposing the state space of each individual test instance in disjoint sub spaces. The decomposition is obtained using four simple filters. A filter is like a decision node in classification trees. It takes as input a state space $\mathcal{S}$ and splits it in $n$ disjoint sub state spaces $\mathcal{S}_{1}, \ldots, \mathcal{S}_{n}$ such that $\mathcal{S}_{1} \cup \ldots \cup \mathcal{S}_{n}=\mathcal{S}$, based on the evaluation of the filter expression for all states $s \in \mathcal{S}$. The first filter evaluates for each state $(\mathbf{z}, j, k)$ the customer class $k$. The second filter evaluates for each state whether or not the DA-rule and the SA-rule propose the same decision. For states where the two rules propose the same decision, we do not decompose further. For all other states, we apply a third and a fourth filter. The third filter considers for each state the associated DA decision and distinguishes between three options: (i) the DA-rule chooses the central warehouse, (ii) the DA-rule chooses a local warehouse with exactly one item on stock, and (iii) the DA-rule chooses a local warehouse with more than one item on stock. The fourth filter is similar to the third, but considers the SA decision instead of the DA decision. These four filters decompose the state space of each test instance into $(3 \times 1 \times 1)+(3 \times 1 \times(9-1))=27$ disjoint sub spaces.

By solving the balance equations for the Markov chain induced by the DA-rule, we obtain the fraction of all decision events that belong to each of these 27 sub spaces. This provides valuable information on when and how often the DA-rule and the SA-rule propose different decisions. When the two rules propose different decisions, we investigate how the DA-rule rates the decision proposed by the SA-rule. For this purpose, we introduce the variable $q(x, y, \mathbf{z}, j, k)=\left[C_{y j k}^{f}+h^{D A}\left(\mathbf{z}-\mathbf{e}_{y}, 0,0\right)\right]-\left[C_{x j k}^{f}+h^{D A}\left(\mathbf{z}-\mathbf{e}_{x}, 0,0\right)\right]$. We can interpret $q(x, y, \mathbf{z}, j, k)$ as the cost difference (according to the DA-rule) when fulfilling a demand from customer region $j$ and customer class $k$ from warehouse $x$ instead of warehouse $y$ if the actual stock level vector is $\mathbf{z}$. We define the cost difference $q(\mathcal{S})$ over a set of states $\mathcal{S}$ as the weighted sum of all $q\left(a^{D A}(\mathbf{z}, j, k), a^{S A}(\mathbf{z}, j, k), \mathbf{z}, j, k\right)$ with $(\mathbf{z}, j, k) \in \mathcal{S}$. Let $\pi(\mathbf{z}, j, k)$ denote the steady state probability of state $(\mathbf{z}, j, k)$ under the DA-rule. Then, we get:

$$
q(\mathcal{S})=\frac{\sum_{(\mathbf{z}, j, k) \in \mathcal{S}} \pi(\mathbf{z}, j, k) q\left(a^{D A}(\mathbf{z}, j, k), a^{S A}(\mathbf{z}, j, k), \mathbf{z}, j, k\right)}{\sum_{(\mathbf{z}, j, k) \in S} \pi(\mathbf{z}, j, k)}
$$

In Table 6 we have shown the average over all 2430 test instances of the decision event probabilities and $q(\mathcal{S})$ for all 27 sub spaces. In line with previous notation, $a^{D A}\left(a^{S A}\right)$ represents the warehouse selected by 


\begin{tabular}{|c|c|c|c|c|c|c|}
\hline \multirow[t]{2}{*}{ Subset No. } & \multicolumn{4}{|c|}{ State space decomposition } & \multirow[t]{2}{*}{ Event probability } & \multirow[t]{2}{*}{$q(S)$} \\
\hline & Filter 1 & Filter 2 & Filter 3 & Filter 4 & & \\
\hline 1 & $k=1$ & $a^{D A}=a^{S A}$ & $\mathrm{~N} / \mathrm{A}$ & $\mathrm{N} / \mathrm{A}$ & 0.309 & 0 \\
\hline 2 & . & $a^{D A} \neq a^{S A}$ & $a^{D A}=0$ & $z\left(a^{S A}\right)=1$ & 0 & 414 \\
\hline 3 & . & . & . & $z\left(a^{S A}\right)>1$ & 0 & 249 \\
\hline 4 & . & . & $z\left(a^{D A}\right)=1$ & $a^{S A}=0$ & 0 & N/A \\
\hline 5 & . & . & . & $z\left(a^{S A}\right)=1$ & 0.014 & 49 \\
\hline 6 & . & . & . & $z\left(a^{S A}\right)>1$ & 0.001 & 18 \\
\hline 7 & . & . & $z\left(a^{D A}\right)>1$ & $a^{S A}=0$ & 0 & N/A \\
\hline 8 & . & . & . & $z\left(a^{S A}\right)=1$ & 0.007 & 68 \\
\hline 9 & . & . & . & $z\left(a^{S A}\right)>1$ & 0.002 & 27 \\
\hline 10 & $k=2$ & $a^{D A}=a^{S A}$ & N/A & N/A & 0.276 & 0 \\
\hline 11 & . & $a^{D A} \neq a^{S A}$ & $a^{D A}=0$ & $z\left(a^{S A}\right)=1$ & 0.007 & 321 \\
\hline 12 & . & . & . & $z\left(a^{S A}\right)>1$ & 0.000 & 202 \\
\hline 13 & . & . & $z\left(a^{D A}\right)=1$ & $a^{S A}=0$ & 0 & N/A \\
\hline 14 & . & . & . & $z\left(a^{S A}\right)=1$ & 0.031 & 55 \\
\hline 15 & . & . & . & $z\left(a^{S A}\right)>1$ & 0.001 & 19 \\
\hline 16 & . & . & $z\left(a^{D A}\right)>1$ & $a^{S A}=0$ & 0 & N/A \\
\hline 17 & . & . & . & $z\left(a^{S A}\right)=1$ & 0.015 & 76 \\
\hline 18 & . & . & . & $z\left(a^{S A}\right)>1$ & 0.003 & 28 \\
\hline 19 & $k=3$ & $a^{D A}=a^{S A}$ & N/A & N/A & 0.264 & 0 \\
\hline 20 & . & $a^{D A} \neq a^{S A}$ & $a^{D A}=0$ & $z\left(a^{S A}\right)=1$ & 0.027 & 850 \\
\hline 21 & . & . & . & $z\left(a^{S A}\right)>1$ & 0.001 & 608 \\
\hline 22 & . & . & $z\left(a^{D A}\right)=1$ & $a^{S A}=0$ & 0 & N/A \\
\hline 23 & . & . & . & $z\left(a^{S A}\right)=1$ & 0.024 & 49 \\
\hline 24 & . & . & . & $z\left(a^{S A}\right)>1$ & 0.001 & 18 \\
\hline 25 & . & . & $z\left(a^{D A}\right)>1$ & $a^{S A}=0$ & 0 & N/A \\
\hline 26 & . & . & . & $z\left(a^{S A}\right)=1$ & 0.014 & 72 \\
\hline 27 & . & . & . & $z\left(a^{S A}\right)>1$ & 0.002 & 27 \\
\hline
\end{tabular}

Table 6: DA-rule characterization

the DA (SA) rule, and $z(i)$ represents the actual stock level at warehouse $i$. From Table 6 we learn that on average for $100 \% \times(0.309+0.276+0.264)=84.9 \%$ of all part requests the DA-rule and the SA-rule propose the same allocation decision. From subset 2 and 3 we learn that the DA-rule will only execute an emergency delivery for 2 hours demand if there is no other option. Subsets 11 and 12, and 20 and 21 indicate that for 4 hours demand, and in particular for 8 hours demand, this is different; here the DA-rule sometimes decides to use an emergency delivery instead of a regular delivery from a local warehouse with exactly one item on stock. The explanation is that the DA-rule anticipates events where a new 2 hours demand arrives before 
the current part is being replenished and finds all nearby local warehouses out of stock. This is the most expensive event that can occur. Consequently, it might be beneficial in some circumstances to minimize the chance of occurrence of such an event by fulfilling a part request from the ample stock central warehouse and not from a local warehouse.

The figures for subsets 8,17 , and 26 show that it also happens that the DA-rule decides to deliver from a local warehouse with more than one item on stock whereas the SA-rule decides to take away that last stock item of a local warehouse. Summarizing we can say that the main difference between the DA-rule and the SA-rule is that the DA-rule is more reluctant to take away the last item at a local warehouse and less reluctant to use emergency deliveries from the central warehouse. The $q(\mathcal{S})$ values show that the DA-rule sees the biggest benefits in fulfilling some part requests with a maximum response time of 8 hours from the central warehouse instead of a local warehouse.

\subsection{Experiment II}

In this experiment we compare the DA-rule and the SA-rule on a test bed containing problem instances of real-life size. The test bed has been defined in Subsection 5.1. For each of the 3240 test instances, we have calculated the total cost, the regular delivery cost, the emergency delivery cost, and the penalty cost for the DA-rule, and the SA-rule. In contrast to Experiment I, we cannot evaluate the performance of the DA-rule and the SA-rule analytically and thus we move to discrete event simulation. See Subsection 5.4.1 for the details of how the simulation has been set up.

The average cost reduction over all 3240 test instances when switching from the SA-rule to the DA-rule is $7.9 \%$. When leaving out all test instances with $\boldsymbol{\Gamma}=(0.98,098,0.98)$ (this parameter value is not present in Experiment I), the average cost reduction is even 9.7\%. This is more than $30 \%$ bigger than the average cost reduction in Experiment I (7.3\%) and a clear indication that the DA-rule scales well. To investigate how the cost savings depend on the various problem characteristics, we have created a box diagram for each parameter in the factorial design. The box diagrams are shown in Figures 3(a)-3(f) and show the median, the $25 \%$ and $75 \%$ percentiles, and the minimum and maximum cost reductions.

The Figures 3(a)-3(f) give a similar picture as the results in Experiment I (cf. Table 4). We see that the cost reduction for test instances with region indicator $R=r_{2}$ (emergency delivery time of 8 hours) is bigger than the cost reduction for test instances with region indicator $R=r_{1}$ (emergency delivery time of 4 hours). We also see that the cost reduction gets bigger if the service area gets smaller. Figure 3(c) shows no clear correlation between the cost reduction and the weight vector $\mathbf{w}$ of the customer classes. Figures 3(d) to 3(f) however, show a strong correlation between the cost savings and the relative network demand $\phi$, the penalty cost vector $\mathbf{C}^{\mathbf{p}}$, and the target time-based fill rate vector $\boldsymbol{\Gamma}$, just as in Experiment I.

All together, switching from the SA-rule to the DA-rule seems in particular beneficial in situations 


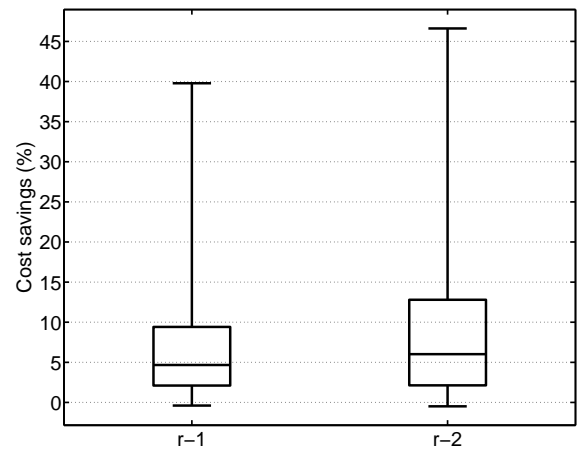

(a) Region indicator $(R)$

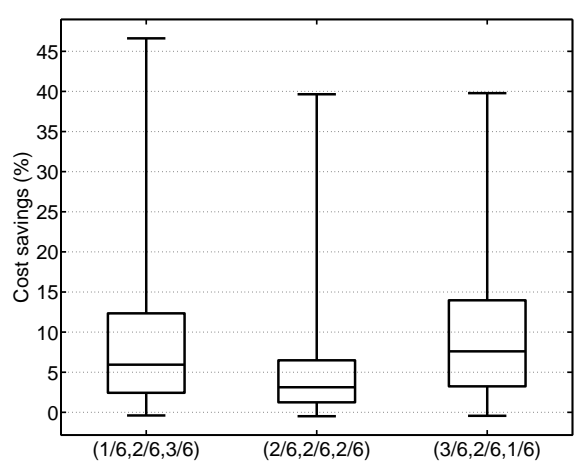

(c) Customer class fractions $(\mathbf{w})$

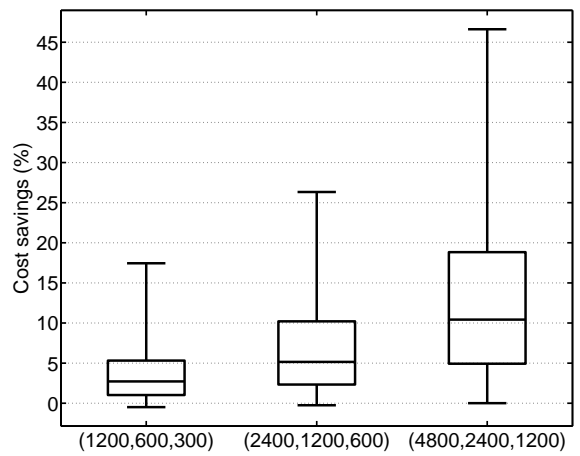

(e) Penalty cost vector $\left(\mathbf{C}^{\mathbf{p}}\right)$

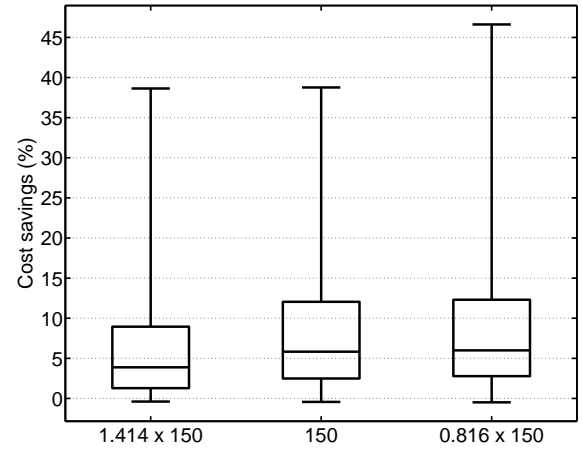

(b) Length service area unit (l)

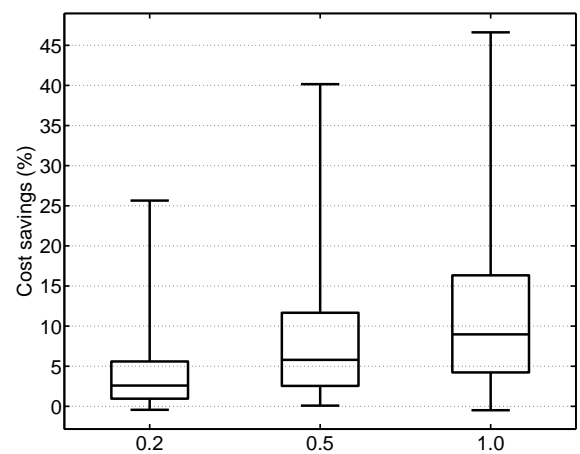

(d) Relative network demand ( $\phi$ )

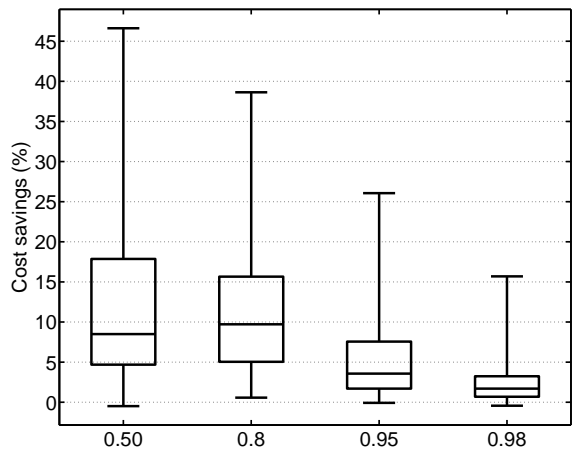

(f) Target time-based fill rate ( $\mathbf{\Gamma})$

Figure 3: Experiment II: Box diagrams for parameters in factorial design

where: (i) contract violations are expensive and occur frequently, (ii) emergency shipments involve high delivery and/or penalty cost, and (iii) the probability of multiple demands within one replenishment lead time is relatively high. In all these situations, the SA-rule suffers from the weakness that it does not anticipate future stockouts and the contract violations and penalty costs this may cause. 


\subsubsection{Discrete event simulation}

Here, we describe how we have set up our discrete event simulations. Each simulation run is divided in 21 sub-runs of 5,000 part failure events each. The first sub-run is used as a warming-up period. For all other 20 sub-runs we calculate the average annual total cost. From these 20 samples we calculate the sample mean and the coefficient of variation. If the coefficient of variation is smaller than $0.01 / 2$ the simulation is terminated. Otherwise, we double the number of part failure events we want to simulate and merge the current 20 sub-runs into 10 new ones by merging sub-runs 1 with 2,3 with $4, \ldots$, and 19 with 20 . Under some mild conditions this methods guarantees that the simulated annual total cost does not differ more than $1 \%$ from its expected value with a probability of more than $95 \%$. This method is called the method of non-overlapping batch means (NOBM). For a comprehensive description of NOBM we refer the reader to Steiger \& Wilson (2001).

\section{Conclusions}

We conclude by summarizing our main results. We developed a dynamic rule for allocating available network stock to real-time part requests in a single echelon multi location spare parts network with multiple customer classes where part requests can often be fulfilled from more than one warehouse within the service deadline. Our dynamic allocation rule is a one-step lookahead policy that approximates the optimal relative cost with an estimate of the relative cost under a static allocation rule.

First, we showed on a test bed with small problem instances that the optimality gap of our dynamic allocation rule is small ( $1.6 \%$ on average). This is much smaller than the optimality gap of a simple but widely used static allocation rule (9.6\% on average). We also showed that on problem instances of real-life size, the dynamic allocation rule achieves average cost savings of $7.9 \%$ in comparison to the static allocation rule. This indicates that the dynamic allocation rule scales very well. Second, we showed that the dynamic allocation rule in particular outperforms the static allocation rule when contract violations are expensive and occur frequently, emergency shipments involve high delivery and/or penalty cost, and the probability of multiple demands within one replenishment lead time is relatively high. Third, we characterized our dynamic allocation rule and showed that it mainly differs from the static allocation rule in situations where the static allocation rule selects a warehouse with only one item on stock, and the actual part request has a relatively high maximum response time. In particular, we showed that switching from the greedy static allocation rule to the dynamic allocation rule with (limited) lookahead changes the role of emergency deliveries from a tool of last resort to a tool that is actively used to avoid stock level reductions at local warehouses in situations where this is considered undesirable.

For future research, it is relevant to investigate the impact of demand forecast errors on the performance 
of the DA-rule. Another interesting direction for future research is the optimization of base stock levels under a dynamic allocation rule like the one presented in this paper.

\section{References}

Alfredsson, P., \& Verrijdt, J. (1999). Modeling emergency supply flexibility in a two-echelon inventory system. Management Science, 45, 1416-1431.

Axsäter, S. (1990). Modelling emergency lateral transshipments in inventory systems. Management Science, 36, 1329-1338.

Axsäter, S. (2003). A new decision rule for lateral transshipments in inventory systems. Management Science, 49, 1168-1179.

Bertsekas, D. (2007). Dynamic Programming and Optimal Control 3rd Edition, Volume II. Athena Scientific.

Jalil, M. (2011). Customer Information Driven After Sales Service Management: Lessons from Spare Parts Logistics. ERIM PhD Series Research in Management. Erasmus University Rotterdam.

Kranenburg, A., \& Van Houtum, G. (2009). A new partial pooling structure for spare parts networks. European Journal of Operational Research, 199, 908-921.

Kukreja, A., Schmidt, C., \& Miller, D. (2001). Stocking decisions for low-usage items in a multilocation inventory system. Management Science, 47, 1371-1383.

Kutanoglu, E. (2008). Insights into inventory sharing in service parts logistics systems with time-based service levels. Computers $\mathcal{E}$ Industrial Engineering, 54, 341-358.

Minner, S., Silver, E., \& Robb, D. (2003). An improved heuristic for deciding on emergency transshipments. European Journal of Operational Research, 148, 384-400.

Paterson, C., Kiesmuller, G., Teunter, R., \& Glazebrook, K. (2011). Inventory models with lateral transshipments: A review. European Journal of Operational Research, 210, 125-136.

Reijnen, I., Tan, T., \& Van Houtum, G. (2009). Inventory planning for spare parts networks with delivery time requirements. Beta working paper 280. Eindhoven University of Technology, The Netherlands.

Steiger, N., \& Wilson, J. (2001). Convergence properties of the batch means method for simulation output analysis. INFORMS Journal on Computing, 13, 277-293.

Teunter, R., \& Klein Haneveld, W. (2008). Dynamic inventory rationing strategies for inventory systems with two demand classes, poisson demand and backordering. European Journal of Operational Research, 190, 156-178.

Topkis, D. (1968). Optimal ordering and rationing policies in a nonstationary dynamic inventory model with $\mathrm{n}$ demand classes. Management Science, 15, 160-176.

Van Wijk, A., Adan, I., \& van Houtum, G. (2011). Optimal lateral transshipment policy for a two location inventory problem. European Journal of Operational Research, 218, 624-635.

Veinott Jr, A. (1965). Optimal policy for a multi-product, dynamic, nonstationary inventory problem. Management Science, 12, 206-222.

Wong, H., van Houtum, G., Cattrysse, D., \& Van Oudheusden, D. (2005). Simple, efficient heuristics for multi-item multi-location spare parts systems with lateral transshipments and waiting time constraints. Journal of the Operational Research Society, 56, $1419-1430$. 


\section{Appendix A. Calculation of base stock levels}

In this appendix, we formally state the method for calculating a base stock level vector $\mathbf{S}$ from a target time-based fill rates vector $\boldsymbol{\Gamma}$. It consists of two parts. Algorithm 2 approximates the average cost per time unit and the time-based fill rates for given base stock level vectors. In Algorithm 3 we use this evaluation procedure to find a base stock level vector such that the (approximated) time-based fill rates exceed the target time-based fill rates, and (approximated) total cost are low.
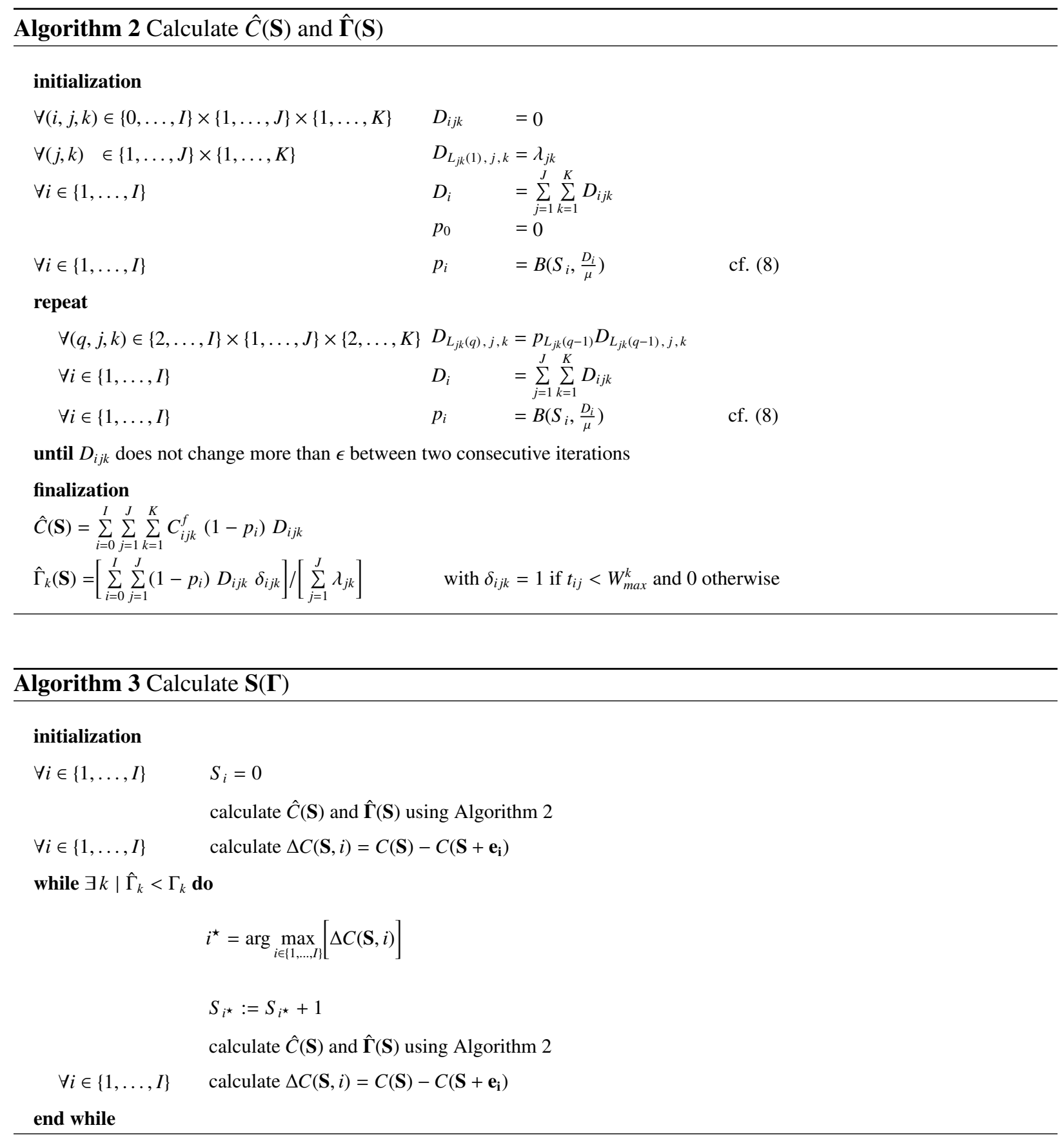
Working Papers Beta 2009 - 2012

nr. Year Title

3772012 Dynamic demand fulfillment in spare parts networks with multiple customer classes

3762012 Improving the performance of sorter systems by scheduling inbound containers

3752012 Strategies for dynamic appointment making by container terminals

MyPHRMachines: Lifelong Personal Health 3742012 Records in the Cloud

Service differentiation in spare parts supply 3732012 through dedicated stocks

Spare parts inventory pooling: how to share 3722012 the benefits

3712012 Condition based spare parts supply

3702012 Using Simulation to Assess the Opportunities of Dynamic Waste Collection

3692012 Aggregate overhaul and supply chain planning for rotables

3682012

Operating Room Rescheduling

Switching Transport Modes to Meet Voluntary 3672011 Carbon Emission Targets

On two-echelon inventory systems with Poisson 3662011 demand and lost sales

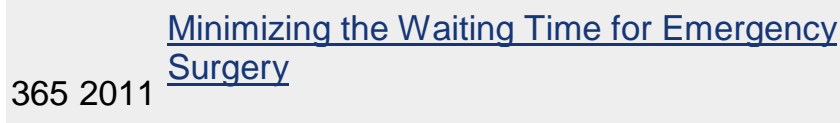

Minimizing the Waiting Time for Emergency 3652011 Surgery

3642011 Vehicle Routing Problem with Stochastic Travel Times Including Soft Time Windows and Service
Author(s)

H.G.H. Tiemessen, M. Fleischmann,

G.J. van Houtum, J.A.E.E. van Nunen,

E. Pratsini

K. Fikse, S.W.A. Haneyah, J.M.J.

Schutten

Albert Douma, Martijn Mes

Pieter van Gorp, Marco Comuzzi

E.M. Alvarez, M.C. van der Heijden, W.H.M. Zijm

Frank Karsten, Rob Basten

X.Lin, R.J.I. Basten, A.A. Kranenburg, G.J. van Houtum

Martijn Mes

J. Arts, S.D. Flapper, K. Vernooij

J.T. van Essen, J.L. Hurink, W. Hartholt, B.J. van den Akker

Kristel M.R. Hoen, Tarkan Tan, Jan C. Fransoo, Geert-Jan van Houtum

Elisa Alvarez, Matthieu van der Heijden J.T. van Essen, E.W. Hans, J.L. Hurink, A. Oversberg

Duygu Tas, Nico Dellaert, Tom van Woensel, Ton de Kok 
A New Approximate Evaluation Method for Two3632011 Echelon Inventory Systems with Emergency Shipments

3622011 Approximating Multi-Objective Time-Dependent Optimization Problems

Branch and Cut and Price for the Time

3612011 Dependent Vehicle Routing Problem with Time Window

3602011 Analysis of an Assemble-to-Order System with Different Review Periods

3592011 Interval Availability Analysis of a Two-Echelon Multi-Item System

Carbon-Optimal and Carbon-Neutral Supply

3582011 Chains

Generic Planning and Control of Automated

3572011 Material Handling Systems: Practical

Requirements Versus Existing Theory

3562011

Last time buy decisions for products sold under warranty

3552011

Spatial concentration and location dynamics in logistics: the case of a Dutch provence

3542011 Identification of Employment Concentration Areas

3532011 BOMN 2.0 Execution Semantics Formalized as Graph Rewrite Rules: extended version

3522011 Resource pooling and cost allocation among independent service providers

3512011 A Framework for Business Innovation Directions

3502011 The Road to a Business Process Architecture: An Overview of Approaches and their Use
Erhun Özkan, Geert-Jan van Houtum, Yasemin Serin

Said Dabia, El-Ghazali Talbi, Tom Van Woensel, Ton de Kok

Said Dabia, Stefan Röpke, Tom Van Woensel, Ton de Kok

A.G. Karaarslan, G.P. Kiesmüller, A.G. de Kok

Ahmad Al Hanbali, Matthieu van der Heijden

Felipe Caro, Charles J. Corbett, Tarkan Tan, Rob Zuidwijk

Sameh Haneyah, Henk Zijm, Marco Schutten, Peter Schuur

M. van der Heijden, B. Iskandar

Frank P. van den Heuvel, Peter W. de Langen, Karel $\mathrm{H}$. van Donselaar, Jan C. Fransoo

Frank P. van den Heuvel, Peter W. de Langen, Karel $\mathrm{H}$. van Donselaar, Jan C. Fransoo

Pieter van Gorp, Remco Dijkman

Frank Karsten, Marco Slikker, Geert-Jan van Houtum

E. Lüftenegger, S. Angelov, P. Grefen

Remco Dijkman, Irene Vanderfeesten, Hajo A. Reijers 
3492011 Effect of carbon emission regulations on transport mode selection under stochastic demand

3482011 An improved MIP-based combinatorial approach for a multi-skill workforce scheduling problem

An approximate approach for the joint problem of

3472011 level of repair analysis and spare parts stocking

Joint optimization of level of repair analysis and

3462011 spare parts stocks

3452011 Inventory control with manufacturing lead time flexibility

$3442011 \frac{\text { Analysis of resource pooling games via a new }}{\text { extenstion of the Erlang loss function }}$ 3432011

Vehicle refueling with limited resources

Optimal Inventory Policies with Non-stationary

Supply Disruptions and Advance Supply

3422011 Information

Redundancy Optimization for Critical

3412011

Components in High-Availability Capital Goods

Analysis of a two-echelon inventory system with 3392010 two supply modes

Analysis of the dial-a-ride problem of Hunsaker and Savelsbergh

3382010

Attaining stability in multi-skill workforce

scheduling

3352010

Flexible Heuristics Miner (FHM)

3342010

An exact approach for relating recovering

3332010 surgical patient workload to the master surgical schedule

Efficiency evaluation for pooling resources in 3322010 health care
K.M.R. Hoen, T. Tan, J.C. Fransoo

G.J. van Houtum

Murat Firat, Cor Hurkens

R.J.I. Basten, M.C. van der Heijden, J.M.J. Schutten

R.J.I. Basten, M.C. van der Heijden, J.M.J. Schutten

Ton G. de Kok

Frank Karsten, Marco Slikker, Geert-Jan van Houtum

Murat Firat, C.A.J. Hurkens, Gerhard J. Woeginger

Bilge Atasoy, Refik Güllü, TarkanTan

Kurtulus Baris Öner, Alan Scheller-Wolf Geert-Jan van Houtum

Joachim Arts, Gudrun Kiesmüller

Murat Firat, Gerhard J. Woeginger

Murat Firat, Cor Hurkens

A.J.M.M. Weijters, J.T.S. Ribeiro

P.T. Vanberkel, R.J. Boucherie, E.W. Hans, J.L. Hurink, W.A.M. van Lent, W.H. van Harten

Peter T. Vanberkel, Richard J. Boucherie, Erwin W. Hans, Johann L. Hurink, Nelly 
Litvak

The Effect of Workload Constraints in

3312010 Mathematical Programming Models for

Production Planning

M.M. Jansen, A.G. de Kok, I.J.B.F. Adan

$3302010 \frac{\text { Using pipeline information in a multi-echelon }}{\text { spare parts inventory system }}$

Christian Howard, Ingrid Reijnen, Johan

Marklund, Tarkan Tan

Reducing costs of repairable spare parts supply 3292010 systems via dynamic scheduling

Identification of Employment Concentration and 3282010 Specialization Areas: Theory and Application

F.P. van den Heuvel, P.W. de Langen, K.H. van Donselaar, J.C. Fransoo

A combinatorial approach to multi-skill workforce 3272010 scheduling

Murat Firat, Cor Hurkens

3262010 Stability in multi-skill workforce scheduling

Murat Firat, Cor Hurkens, Alexandre Laugier

Maintenance spare parts planning and control: A framework for control and agenda for future

3252010 research

M.A. Driessen, J.J. Arts, G.J. v. Houtum, W.D. Rustenburg, B. Huisman

Near-optimal heuristics to set base stock levels

3242010 in a two-echelon distribution network

R.J.I. Basten, G.J. van Houtum

Inventory reduction in spare part networks by

3232010 selective throughput time reduction

M.C. van der Heijden, E.M. Alvarez,

J.M.J. Schutten

The selective use of emergency shipments for

3222010

service-contract differentiation

Heuristics for Multi-Item Two-Echelon Spare

Parts Inventory Control Problem with Batch

3212010 Ordering in the Central Warehouse

B. Walrave, K. v. Oorschot, A.G.L.

Romme

Preventing or escaping the suppression

3202010

mechanism: intervention conditions

Nico Dellaert, Jully Jeunet.

Hospital admission planning to optimize major 3192010

resources utilization under uncertainty

Minimal Protocol Adaptors for Interacting

R. Seguel, R. Eshuis, P. Grefen.

E.M. Alvarez, M.C. van der Heijden, W.H. Zijm 


\section{Services}

3182010

Teaching Retail Operations in Business and Engineering Schools

3172010

Design for Availability: Creating Value for

Manufacturers and Customers

3162010

Transforming Process Models: executable rewrite rules versus a formalized Java program

3152010 Getting trapped in the suppression of exploration: A simulation model

3142010

A Dynamic Programming Approach to MultiObjective Time-Dependent Capacitated Single 3132010 Vehicle Routing Problems with Time Windows

2010

Tales of a So(u)rcerer: Optimal Sourcing

3122010 Decisions Under Alternative Capacitated Suppliers and General Cost Structures

In-store replenishment procedures for perishable

3112010 inventory in a retail environment with handling costs and storage constraints

3102010 The state of the art of innovation-driven business models in the financial services industry

3092010 Design of Complex Architectures Using a Three Dimension Approach: the CrossWork Case

3082010 Effect of carbon emission regulations on transport mode selection in supply chains

$3072010 \frac{\text { Interaction between intelligent agent strategies }}{\text { for real-time transportation planning }}$

3062010 Internal Slackening Scoring Methods $3052010 \frac{\text { Vehicle Routing with Traffic Congestion and }}{\text { Drivers' Driving and Working Rules }}$

3042010 Practical extensions to the level of repair analysis

Ocean Container Transport: An Underestimated

3032010 and Critical Link in Global Supply Chain Performance

Capacity reservation and utilization for a

3022010 manufacturer with uncertain capacity and demand

3002009 Spare parts inventory pooling games
Tom Van Woensel, Marshall L. Fisher, Jan C. Fransoo.

Lydie P.M. Smets, Geert-Jan van Houtum, Fred Langerak.

Pieter van Gorp, Rik Eshuis.

Bob Walrave, Kim E. van Oorschot, A. Georges L. Romme

S. Dabia, T. van Woensel, A.G. de Kok

Osman Alp, Tarkan Tan

R.A.C.M. Broekmeulen, C.H.M. Bakx

E. Lüftenegger, S. Angelov, E. van der Linden, P. Grefen

R. Seguel, P. Grefen, R. Eshuis

K.M.R. Hoen, T. Tan, J.C. Fransoo, G.J. van Houtum

Martijn Mes, Matthieu van der Heijden, Peter Schuur

Marco Slikker, Peter Borm, René van den Brink

A.L. Kok, E.W. Hans, J.M.J. Schutten, W.H.M. Zijm

R.J.I. Basten, M.C. van der Heijden, J.M.J. Schutten

Jan C. Fransoo, Chung-Yee Lee

Y. Boulaksil; J.C. Fransoo; T. Tan

F.J.P. Karsten; M. Slikker; G.J. van Houtum 
2992009 Capacity flexibility allocation in an outsourced supply chain with reservation

$2982010 \frac{\text { An optimal approach for the joint problem of }}{\text { level of repair analysis and spare parts stocking }}$ Responding to the Lehman Wave: Sales

2972009 Forecasting and Supply Management during the $\underline{\text { Credit Crisis }}$

An exact approach for relating recovering

2962009 surgical patient workload to the master surgical schedule

An iterative method for the simultaneous

2952009 optimization of repair decisions and spare parts stocks

2942009 Fujaba hits the Wall(-e) $2932009 \frac{\text { Implementation of a Healthcare Process in Four }}{\text { Different Workflow Systems }}$

2922009 Business Process Model Repositories Framework and Survey 2912009 Efficient Optimization of the Dual-Index Policy

$2902009 \frac{\text { Hierarchical Knowledge-Gradient for Sequential }}{\text { Sampling }}$ Sampling

Analyzing combined vehicle routing and break

2892009 scheduling from a distributed decision making perspective

2882009 Anticipation of lead time performance in Supply Chain Operations Planning $2872009 \frac{\text { Inventory Models with Lateral Transshipments: A }}{\text { Review }}$ 2862009 Efficiency evaluation for pooling resources in

$2852009 \frac{\text { A Survey of Health Care Models that }}{\text { Encompass Multiple Departments }}$

$2842009 \frac{\text { Supporting Process Control in Business }}{\text { Collaborations }}$

2832009 Inventory Control with Partial Batch Ordering

$2822009 \frac{\text { Translating Safe Petri Nets to Statecharts in a }}{\text { Structure-Preserving Way }}$ Structure-Preserving Way

$2812009 \frac{\text { The link between product data model and }}{\text { process model }}$

$2802009 \frac{\text { Inventory planning for spare parts networks with }}{\text { delivery time requirements }}$

2792009 Co-Evolution of Demand and Supply under
Y. Boulaksil, M. Grunow, J.C. Fransoo

R.J.I. Basten, M.C. van der Heijden, J.M.J. Schutten

Robert Peels, Maximiliano Udenio, Jan C Fransoo, Marcel Wolfs, Tom Hendrikx

Peter T. Vanberkel, Richard J. Boucherie, Erwin W. Hans, Johann L. Hurink, Wineke A.M. van Lent, Wim H. van Harten

R.J.I. Basten, M.C. van der Heijden, J.M.J. Schutten

Pieter van Gorp, Ruben Jubeh, Bernhard Grusie, Anne Keller

R.S. Mans, W.M.P. van der Aalst, N.C.

Russell, P.J.M. Bakker

Zhiqiang Yan, Remco Dijkman, Paul Grefen

Joachim Arts, Marcel van Vuuren, Gudrun Kiesmuller

Martijn R.K. Mes; Warren B. Powell; Peter I. Frazier

C.M. Meyer; A.L. Kok; H. Kopfer; J.M.J. Schutten

Michiel Jansen; Ton G. de Kok; Jan C. Fransoo

Colin Paterson; Gudrun Kiesmuller; Ruud Teunter; Kevin Glazebrook

P.T. Vanberkel; R.J. Boucherie; E.W. Hans; J.L. Hurink; N. Litvak

P.T. Vanberkel; R.J. Boucherie; E.W. Hans; J.L. Hurink; N. Litvak

S. Angelov; K. Vidyasankar; J. Vonk; P. Grefen

O. Alp; W.T. Huh; T. Tan

R. Eshuis

J.J.C.L. Vogelaar; H.A. Reijers

I.C. Reijnen; T. Tan; G.J. van Houtum

B. Vermeulen; A.G. de Kok 


\section{Competition}

Toward Meso-level Product-Market Network

2782010 Indices for Strategic Product Selection and

B. Vermeulen, A.G. de Kok

$(\mathrm{Re})$ Design Guidelines over the Product Life-

Cycle

An Efficient Method to Construct Minimal

R. Seguel, R. Eshuis, P. Grefen

2772009 Protocol Adaptors

$2762009 \frac{\text { Coordinating Supply Chains: a Bilevel }}{\text { Programming Approach }}$

Ton G. de Kok, Gabriella Muratore

$2752009 \frac{\text { Inventory redistribution for fashion products }}{\underline{\text { under demand parameter update }}}$

G.P. Kiesmuller, S. Minner

Comparing Markov chains: Combining

2742009 aggregation and precedence relations applied to A. Busic, I.M.H. Vliegen, A. Scheller-Wolf sets of states

2732009 Separate tools or tool kits: an exploratory study of engineers' preferences

I.M.H. Vliegen, P.A.M. Kleingeld, G.J. van Houtum

An Exact Solution Procedure for Multi-Item Two-

2722009 Echelon Spare Parts Inventory Control Problem with Batch Ordering

Engin Topan, Z. Pelin Bayindir, Tarkan

Tan

Distributed Decision Making in Combined Vehicle Routing and Break Scheduling

C.M. Meyer, H. Kopfer, A.L. Kok, M.

Schutten

Dynamic Programming Algorithm for the Vehicle

2702009 Routing Problem with Time Windows and EC Social Legislation

A.L. Kok, C.M. Meyer, H. Kopfer, J.M.J. Schutten

Remco Dijkman, Marlon Dumas,

2692009 Similarity of Business Process Models: Metics

Boudewijn van Dongen, Reina Kaarik, Jan Mendling

$2672009 \frac{\text { Vehicle routing under time-dependent travel }}{\text { times: the impact of congestion avoidance }}$

A.L. Kok, E.W. Hans, J.M.J. Schutten

2662009 Restricted dynamic programming: a flexible

J. Gromicho; J.J. van Hoorn; A.L. Kok; J.M.J. Schutten;

Working Papers published before 2009 see: http://beta.ieis.tue.nl 